
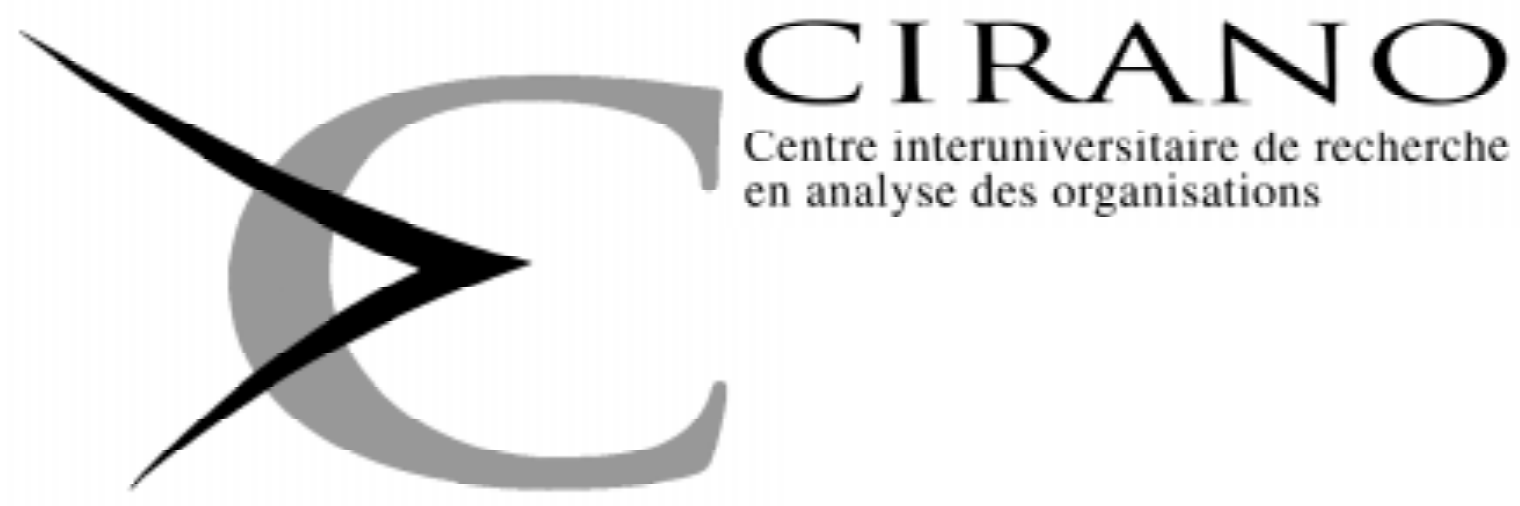

Centre interuniversitaire de recherche en analyse des organisations

Série Scientifique

Scientific Series

No 94s-20
SEQUENTIAL LOCATION
EQUILIBRIA UNDER
INCOMPLETE INFORMATION
Marcel Boyer, Jean-Jacques Laffont,
Philippe Mahenc, Michel Moreaux

Montréal

Décembre 1994 


\section{CIRANO}

Le CIRANO est une corporation privée à but non lucratif constituée en vertu de la Loi des compagnies du Québec. Le financement de son infrastructure et de ses activités de recherche provient des cotisations de ses organisations-membres, d'une subvention d'infrastructure du ministère de l'Industrie, du Commerce, de la Science et de la Technologie, de même que des subventions et mandats obtenus par ses équipes de recherche. La Série Scientifique est la réalisation d'une des missions que s'est données le CIRANO, soit de développer l'analyse scientifique des organisations et des comportements stratégiques.

CIRANO is a private non-profit organization incorporated under the Québec Companies Act. Its infrastructure and research activities are funded through fees paid by member organizations, an infrastructure grant from the Ministere de l'Industrie, du Commerce, de la Science et de la Technologie, and grants and research mandates obtained by its research teams. The Scientific Series fulfils one of the missions of CIRANO: to develop the scientific analysis of organizations and strategic behaviour.

\section{Les organisations-partenaires / The Partner Organizations}

-Ministère de l'Industrie, du Commerce, de la Science et de la Technologie. -École des Hautes Études Commerciales.

-École Polytechnique.

-Université de Montréal.

-Université Laval.

-McGill University.

-Université du Québec à Montréal.

-Bell Québec.

-La Caisse de dépôt et de placement du Québec.

-Hydro-Québec.

-Banque Laurentienne du Canada.

-Fédération des caisses populaires de Montréal et de l'Ouest-du-Québec.

Ce document est publié dans l'intention de rendre accessible les résultats préliminaires de la recherche effectuée au CIRANO, afin de susciter des échanges et des suggestions. Les idées et les opinions émises sont sous l'unique responsabilité des auteurs, et ne représentent pas

nécessairement les positions du CIRANO ou de ses partenaires.

This paper presents preliminary research carried out at CIRANO and aims to encourage discussion and comment. The observations and viewpoints expressed are the sole responsibility of the authors. They do not necessarily represent positions of CIRANO or its partners.

ISSN 1198-8177 


\title{
Sequential location equilibria under incomplete information*
}

\author{
by \\ Marcel Boyer ${ }^{1}$ \\ Jean-Jacques Laffont ${ }^{2}$ \\ Philippe Mahenc ${ }^{3}$ \\ Michel Moreaux ${ }^{4}$
}

\footnotetext{
${ }^{1}$ Stephen A. Jarislowsky Professor of Technology and International Competition, École Polytechnique de Montral; Département de Sciences Economiques, CRDE and CIRANO [Université de Montréal, C.P.6128, Montréal, Canada H3C 3J7]

${ }^{2}$ Institut Universitaire de France; GREMAQ and IDEI [Université de Toulouse I, Place Anatole-France, F-31042 Toulouse, France]

${ }^{3}$ GREMAQ, [Université de Toulouse I, Place Anatole-France, F-31042 Toulouse, France]

${ }^{4}$ GREMAQ and IDEI [Université de Toulouse I, Place Anatole-France, F-31042 Toulouse, France]
}

* We would like to thank an anonymous referee for very helpful comments and also the participants in seminars at GREQE (Marseille), Université de Montréal, UBC, HEC (Paris), in the Location Theory session of the World Congress of the Econometric Society (Barcelona) and in the Game Theory Conference at the University of Western Ontario for their comments. We remain, of course, solely responsible for the content of this paper. Financial support from FCAR (Québec), SSHRCC (Canada) and CNRS (France) is gratefully acknowledged. 
Summary. We consider a simple model of incomplete information in location theory. Two firms compete in a two stage framework: a sequential location stage and a price competition stage. Firm 1 knows both its own constant marginal cost technology and that of Firm $2 \Gamma$ whereas the latter has incomplete information about firm 1's technology. The location stage turns out to be a monotonic signaling game and the unique D1 equilibrium is a pure strategy separating equilibrium if firm 1's cost advantage is below some bound $\Gamma$ and otherwise a pooling equilibrium if the prior probability that Firm 1 is of the low cost type is high Tor a semi-pooling equilibrium if it is low. This surprising result is due to the fact that the location gap between the two types of Firm 1 is bounded because of natural economic reasons Twhich may prevent the separation of the two types. Hence incomplete information matters: the equilibrium locations differ quite significantly from the full information equilibrium locations.

Résumé. Nous proposons ici un modèle simple d’information incomplète en théorie de la localisation. Deux entreprises s'affrontent dans un contexte à deux étapes: une étape de localisation séquentielle et une étape de concurrence en prix. La firme 1 connaît sa technologie à coût marginal constant et celle de la firme 2 mais cette dernière n'a qu'une information imparfaite de la technologie de sa concurrente. La concurrence de première étape s'avère être un jeu de signal monotone et l'équilibre D1 unique est un équilibre séparateur en stratégies pures si l'avantage de cô̂t de la firme 1 est relativement faible et sinon un équilibre mélangeant Tsi la probabilité a priori que la firme 1 soit de type coût faible est élevéeГou un équilibre semi-mélangeant ssi cette probabilité est faible. Ce résultat surprenant est dû au fait que l'écart de localisation entre les firmes est naturellement bornéГce qui peut empêcher la séparation des types. Ainsi les localisations d'équilibre d'information incomplète diffèrent significativement des localisations d'équilibre d'information complète. 


\section{INTRODUCTION}

One important aspect of spatial competition has been neglected so far by location theorists $\Gamma$ namely the incompleteness of the information structures. It is our objective in this paperTdeveloping an analysis only sketched in [1] $\Gamma$ to fully characterize the set of perfect Bayesian equilibria when the source of incomplete information pertains to one entrepreneur's production conditions $\Gamma$ namely cost $\Gamma$ which are unobserved by the competitor.

We consider a spatial competition model fairly simple and standard but capable of addressing such a complex issue as incomplete information in location theory. Two firms compete in a two stage framework: a long run first stage of sequential location choices and a short run second stage of simultaneous choices of delivered price schedules made once the locations chosen are observed. Firm 1 is assumed to have a superior information structure in the following sense. It knows both its own technology and that of Firm 2 Twhereas the latter knows its own technology and has incomplete information about what the first mover firm's technology is. The location space is represented by an interval over which there is a continuum of consumers uniformly distributed. This way of modeling spatial competition under incomplete information may be justified as follows. First Tlocation choices are typically long run decisions $\Gamma$ while pricing decisions are typically short run decisions. It is therefore reasonable to model competition over both locations and prices as a two stage competition $\Gamma$ the second stage or short run stage coming into play once the long run decisions have been made and observed by both firms. Modeling the space of locations as a linear segment stems from the fact that in the alternative basic space used in location theory That is $\Gamma$ a circular market with a uniform density of identical consumers and transportation costs depending only on the distance between the consumer and the firm the choice of location by the informed firm cannot provide useful information to the uninformed firm $\Gamma$ since any location is a perfect replication of any other one (see [5] $[$ [9] $\Gamma$ and [10] for recent surveys). To make the circular model amenable to signaling possibilities Tone could make the distribution of consumers non-uniform. But for analytical purposes Tit would then become isomorphic to the line segment. Hence $\Gamma$ the segment with a uniform density of identical consumers is the simplest spatial structure allowing signaling. We model the short run pricing competition as a Bertrand competition on delivered price schedules for two reasons. First Tit allows us to avoid the problem of existence of equilibrium encountered in the case of mill pricing (see [4]) and to concentrate our efforts on the incomplete information in the long run stage and second $\Gamma$ it is a non-negligible aspect of many empirical situations. Lederer and Hurter [11] refer to such price schedules as discriminatory pricing through the absorption of transportation costs; they claim it is typicalГin oligopolistic markets of goods with low value relative to the transportation costs and low price elasticity of demand such as cement $\Gamma$ plywood $\Gamma$ fertilizer $\Gamma$ sugarTetc. On competition in delivered price schedules $\Gamma$ see also $[2] \Gamma[6] \Gamma[7]$ Tand $[13]$. 
The one-sided information structure we considerT namely a better informed first mover who may or may not have a cost advantage on the second mover Trepresents one of many possible incomplete information structures Tother possibilities being $\Gamma$ for instance $\Gamma$ a better informed second mover and a two-sided incomplete information. Making the second mover better informed would rule out the possibility of signaling. The information structure we consider here has the advantage of simplicityTa desirable property for an introduction of incomplete information in location theory situations.

The main results of this paper are as follows. The first stage location game turns out to be a monotonic signaling game as defined by Cho and Sobel [3] for which equilibrium refinement D1 selects a unique perfect Bayesian equilibrium. We will be able to characterize the type of D1 equilibrium arising under all possible values of the parameters of the model namely the cost advantage of the first mover and the priors. We will show that when the cost differential is below some bound The only D1 equilibrium is a pure strategy separating equilibrium. But for values of the cost discrepancy above this bound the D1 equilibrium will not be a separating equilibrium. This might seem surprising at first sight because one might have expected that for large cost differences $\Gamma$ it would be easier for the low cost first mover to differentiate itself from its high cost twin. But in a spatial context such as the present one $\mathrm{T}$ the location gap between the two types of Firm 1 is bounded because of natural economic reasons 5 preventing the separation of the two types. Hence the equilibria robust to D1 may be either pooling equilibria or semi-pooling equilibria according to whether the prior probability that Firm 1 is of the low cost type is high or low. HenceГincomplete information matters: the equilibrium locations so predicted may be quite different from the full information equilibrium locations. In [1] we examined $\Gamma$ using the same model $\Gamma$ the case in which the firms plays only pure strategies $\Gamma$ thus excluding those equilibria which are intrinsically mixed strategy equilibria such as the semi-separating equilibria. We worked with Cho and Kreps' intuitive criterion in order to discriminate among the equilibria. This criterion Tweaker than D1Tfails to eliminate all the pooling equilibria but one. It can be shown also that it is not very efficient in the selection of semi-separating equilibria.

The paper is organized as follows. Section 2 introduces the model. In Section $3 \Gamma$ we give the definitions of the perfect Bayesian equilibrium (PBE). Section 4 presents the D1 equilibrium refinement conceptT the limit posterior probability function and the review of the basic results of Cho and Sobel on strategic stability in monotonic signaling games. We characterize in section 5 the whole set of PBE and identify those which are robust to refinement D1. We briefly conclude in section 6 .

\section{THE MODEL}

Let us denote by $i=1,2$ the two competing firms: Firm 1 being the first mover and Firm $2 \Gamma$ the 
follower. Each firm can produce at some constant average (hence marginal) cost the same basic product $\Gamma$ whatever its location. The average production cost of Firm 1 is commonly known to be either $c-\alpha$ with probability $\pi$ or $c$ with the complementary probability $1-\pi$ Twhere $c>1 / 2$ and $\alpha \in(0,1 / 2)$. As for Firm 2 its constant average cost is $c$. Firm 1 knows before choosing its own location which production cost (which type) $\theta \in\{c-\alpha, c\}$ it will have at the production stage. The transportation costs are the same for both firms: each firm must support a cost $d q$ to deliver $q$ units to customers located at a distance $d$ from its own plant. Both firms compete in delivered prices.

Consumers are spread evenly on the interval $[0,1]$ with a density of 1 . Each consumer demands inelastically one unit of the basic good produced by any one of the two firms Tprovided that its delivered price not be higher than some reservation value $r$ Wwhich is the same for all the consumers on the segment line. We will assume that $r>1+c$ so that each firm could get some part of the market the type $\theta$ of Firm 1 Wwhen both are charging delivered prices equal to their full unit costs production plus transportation costs. We will also assume as in [11] that if a consumer is charged the same price by both firms The will then buy from the supplier who makes the highest profit on his demand. Remember that at the production or market stage of the game the true cost of Firm 1 is known. If both firms are making the same profit on its purchase $\Gamma$ then a consumer will buy from either one with an arbitrary probability 5 say $\gamma \in(0,1)$ from Firm 1 . The exact value of $\gamma$ does not play any role in the determination of the equilibrium.

At the first stage of the gameГFirm 1 knowing its proper cost $\theta$ chooses some location on the segment line $[0,1] \Gamma$ which will be denoted by $x_{1} \Gamma$ the distance between the 0 -end of the segment and the plant location. Observing the location of Firm 1 but unaware of the true cost of its competitorTFirm 2 then chooses its own location $x_{2}$ Tagain the distance between the 0 -end of the segment and its plant. After that the true cost of Firm 1 is unveiled $\Gamma$ a fairly standard modeling feature since Milgrom and Roberts [12] and a short cut for a whole revelation process of the true type of Firm 1.

At the second stage of the game $\Gamma$ the two firms move simultaneously $\Gamma$ competing in delivered prices. This second stage is a complete information game. The strategy of Firm $i$ in this game is denoted by $p_{i}(\cdot ; \cdot, \cdot, \cdot)$ Tmeaning that for locations $x_{1}$ and $x_{2}$ and for Firm 1 's type $\theta \Gamma$ Firm $i$ will charge a delivered price $p_{i}\left(x ; x_{1}, x_{2}, \theta\right)$ to a consumer located at $x$. Under the above assumptions $\mathrm{Cit}$ is shown in Hurter and Lederer [11] that the equilibrium of this game is given by:

$$
p_{i}\left(x ; x_{1}, x_{2}, \theta\right)=p\left(x ; x_{1}, x_{2}, \theta\right)=\max \left\{\theta+\left|x-x_{1}\right|, c+\left|x-x_{2}\right|\right\}, i=1,2,
$$

that is Tthe equilibrium strategies are the same for the two firms. Without loss of generality that Firm 1 is located on $[0,1 / 2]$ and Firm 2 on $\left[x_{1}, 1\right]$. Hence at equilibrium the market is split into two areas: Firm 1 sells to those customers located in $\left[0,1 / 2\left(x_{1}+c-\theta+x_{2}\right)\right) \Gamma$ Firm 2 to the consumers located 
in $\left(1 / 2\left(x_{1}+c-\theta+x_{2}\right), 1\right]$ the consumers located precisely at $1 / 2\left(x_{1}+c-\theta+x_{2}\right)$ being distributed between the two firms The percentage that each one gets having no impact on its own profits.

Let $\Pi_{i}\left(x_{1}, x_{2}, \theta\right), i=1,2 \Gamma$ be the profit of Firm $i$ at the equilibrium of the second stage subgame:

$$
\begin{aligned}
& \Pi_{1}\left(x_{1}, x_{2}, \theta\right)=1 / 4\left(x_{2}-x_{1}\right)\left(3 x_{1}+x_{2}\right)+\delta(\theta)\left(1 / 2 \alpha\left(x_{1}+x_{2}\right)+1 / 4 \alpha^{2}\right) \\
& \Pi_{2}\left(x_{1}, x_{2}, \theta\right)=\left(x_{2}-x_{1}\right)\left(1-1 / 4\left(x_{1}+3 x_{2}\right)\right)+\delta(\theta)\left(1 / 2 \alpha\left(x_{1}+x_{2}\right)-\alpha+1 / 4 \alpha^{2}\right)
\end{aligned}
$$

where

$$
\delta(\theta)= \begin{cases}0 & \text { if } \quad \theta=c \\ 1 & \text { if } \theta=c-\alpha\end{cases}
$$

From (2) and (3) Гwe may determine the complete information subgame perfect equilibrium of the whole game. From $\Pi_{2}\left(x_{1}, x_{2}, \theta\right)$ we get $x_{2}\left(x_{1}, \theta\right)$ Tthe best reply location function of Firm $2 \Gamma$ if at the first stage $\Gamma$ the proper cost of Firm 1 is common knowledge:

$$
x_{2}\left(x_{1}, \theta\right)=1 / 3\left(2+x_{1}+\delta(\theta) \alpha\right)
$$

Substituting $x_{2}\left(x_{1}, \theta\right)$ for $x_{2}$ in $\Pi_{1}(\cdot)$ gives $\Pi_{1}$ as a concave function of $x_{1}$ Tand maximizing $\Pi_{1}$ on the interval $[0,1 / 2]$ gives the complete information equilibrium location of Firm 1 as a function of the cost discrepancy $\alpha$ :

$$
x_{1}^{*}=x_{1}(\theta)=\min \{2 / 5+\delta(\theta)(4 / 5 \alpha), 1 / 2\}
$$

hence after substitution in $x_{2}\left(x_{1}, \theta\right)$ :

$$
x_{2}^{*}=x_{2}\left(x_{1}^{*}, \theta\right)=\min \{4 / 5+\delta(\theta)(3 / 5 \alpha), 5 / 6+\delta(\theta)(1 / 3 \alpha)\} .
$$

As the cost advantage of Firm 1 increases Tit moves nearer to the center of the market where it stays for $\alpha \geq 1 / 8$. Also as $\alpha$ increases TFirm 2 moves to the right Tbeing pushed at the extreme 1-end of the market when $\alpha$ approaches $1 / 2$. Should $\alpha$ be greater than $1 / 2$ Then Firm 2 would be driven out of the market. Note however $\Gamma$ that the distance between the two firms first decreases when $\alpha<1 / 8$ and then increases when $\alpha>1 / 8$. But the market area of Firm 1 is always increasing. The border delimiting the two areas is given by:

$$
x_{B}^{*}(\alpha)=\max \{3 / 5(1+2 \alpha), 2 / 3(1+\alpha)\}
$$

The equilibrium profits are: 


$$
\begin{aligned}
\Pi_{1}\left(x_{1}^{*}, x_{2}^{*}, \theta\right)= & (1-\delta(\theta))(1 / 5) \\
& +\delta(\theta)\left[\eta(\alpha)\left(1 / 5(1+2 \alpha)^{2}\right)+(1-\eta(\alpha))\left(1 / 36\left(7+32 \alpha+16 \alpha^{2}\right)\right)\right] \\
\Pi_{2}\left(x_{1}^{*}, x_{2}^{*}, \theta\right)= & (1-\delta(\theta))(3 / 25) \\
& +\delta(\theta)\left[\eta(\alpha)\left(3 / 25(1-3 \alpha)^{2}\right)+(1-\eta(\alpha))\left(1 / 12(1-2 \alpha)^{2}\right)\right]
\end{aligned}
$$

where $\eta(\alpha)$ is defined as:

$$
\eta(\alpha)= \begin{cases}1 & \text { if } \alpha \leq 1 / 8 \\ 0 & \text { otherwise }\end{cases}
$$

\section{THE PERFECT BAYESIAN EQUILIBRIA: DEFINITIONS}

Since at the end of the first stage the true cost of Firm 1 is unveiled and the second stage subgame is solved according to (1) Twe may consider the sole first stage which appears as a signaling game. The signal here is the location choice of Firm 1 from which Firm 2 will try to infer the type of Firm 1 in order to locate itself optimally. We are therefore looking for a perfect Bayesian equilibrium (PBE) of the location choice game. Depending upon the parameters of the problem $\Gamma$ that is $\Gamma$ the prior $\pi$ and the cost discrepancy $\alpha \Gamma$ there may be separating equilibria in which Firm 1's location reveals its type $\Gamma$ pooling equilibria in which the location of Firm 1 is the same for both types and therefore reveals nothing regarding its production cost $\Gamma$ and finallyГsemi-separating equilibria in which at least one type of Firm 1 chooses its location at random $\Gamma$ sometimes revealing its type and sometimes choosing the same location as the other type. However Tin this last case Ccontrary to the pooling caseTFirm 2 gets additional information from observing the location common to both types.

A mixed strategy of Firm 1 is a mapping $\Psi_{1}(\cdot): \Theta \rightarrow \mathcal{M}_{1}$ Twhere $\Theta=\{c-\alpha, c\}$ is the set of types of Firm 1 and $\mathcal{M}_{1}$ the set of probability measures defined on $[0,1 / 2]$. We will denote by $M_{1}(\cdot)$ a cumulative distribution for the location $x_{1}$ of Firm 1 and by $M_{1}(\cdot ; \theta)$ the cumulative selected by $\Psi_{1}(\cdot)$ for $\theta$. In the case of pure strategies Twe simply denote the location decision function of Firm 1 by $x_{1}(\cdot): \Theta \rightarrow X_{1}$ Twhere $X_{1}=[0,1 / 2]$. We proceed similarly for Firm 2. From (5) we know that with complete information the most distant location from the 1 -end of the segment Twhich would be chosen by Firm 2 is $x_{2}=2 / 3$. The incomplete information will not alter this bound Tas shown by equation (12) below. Hence Twe may restrict the support of $\mathcal{M}_{2}$ to the subsegment $[2 / 3,1]$ without loss of generality. A mixed strategy of Firm 2 is a mapping $\Psi_{2}(\cdot): X_{1} \rightarrow \mathcal{M}_{2}$ Twhere $\mathcal{M}_{2}$ is the set of probability measures defined on $X_{2}=[2 / 3,1]$. We will denote by $M_{2}(\cdot)$ a cumulative distribution for the location $x_{2}$ of Firm 2 and by $M_{2}\left(\cdot ; x_{1}\right)$ the cumulative 
distribution selected by $\Psi_{2}(\cdot)$ for $x_{1}$. In the case of pure strategies $\Gamma$ we simply denote the location decision function of Firm 2 by $x_{2}(\cdot): X_{1} \rightarrow X_{2}$. FinallyГwe need conditional distributions giving for each location in $X_{1}$ the posterior probability on $\Theta$ that Firm 2 will use in deciding its location. Let $\mu\left(\cdot \mid x_{1}\right)$ be the posterior probability on $\Theta \Gamma$ given that $x_{1}$ has been observed. For any probability measure $\mathcal{M} \Gamma$ we denote by Supp $\mathcal{M}$ the support of $\mathcal{M}$ Tand for any function $f$ Twe denote by $R f$ the range of $f$.

A PBE is a triplet $\left\{\Psi_{1}^{*}(\cdot), \Psi_{2}^{*}(\cdot), \mu^{*}(\cdot \mid \cdot)\right\}$ such that:

(i) $\forall \theta \in \Theta: \Psi_{1}^{*}(\theta) \in \arg \max _{M_{1}(\cdot)} \int_{X_{1}} \int_{X_{2}} \Pi_{1}\left(x_{1}, x_{2}, \theta\right) d M_{2}^{*}\left(x_{2} ; x_{1}\right) d M_{1}\left(x_{1}\right)$;

(ii) $\forall x_{1} \in X_{1}: \Psi_{2}^{*}\left(x_{1}\right) \in \arg \max _{M_{2}(\cdot)} \int_{X_{2}} \sum_{\theta \in \Theta} \mu^{*}\left(\theta \mid x_{1}\right) \Pi_{2}\left(x_{1}, x_{2}, \theta\right) d M_{2}\left(x_{2} ; x_{1}\right)$;

(iii) $\forall x_{1} \in \cup_{\theta \in \Theta} \operatorname{Supp} \Psi_{1}^{*}(\theta) \Gamma \mu^{*}\left(\cdot \mid x_{1}\right)$ is obtained by applying Bayes' rule to the prior distribution $(\pi, 1-\pi)$ Tgiven that $x_{1}$ has been observed Totherwise $\mu^{*}\left(\cdot \mid x_{1}\right)$ is arbitrary.

For pure strategy equilibria $\left\{x_{1}^{*}(\cdot), x_{2}^{*}(\cdot), \mu^{*}(\cdot \mid \cdot)\right\}$ Twe have:

(i') $\forall \theta \in \Theta: x_{1}^{*}(\theta) \in \arg \max _{x_{1} \in X_{1}} \Pi_{1}\left(x_{1}, x_{2}^{*}\left(x_{1}\right), \theta\right) ;$

(ii') $\forall x_{1} \in X_{1}: x_{2}^{*}\left(x_{1}\right) \in \arg \max _{x_{2} \in X_{2}} \sum_{\theta \in \Theta} \mu^{*}\left(\theta ; x_{1}\right) \Pi_{2}\left(x_{1}, x_{2}, \theta\right)$;

(iii') $\forall x_{1} \in R x_{1}^{*}(\cdot) \Gamma \mu^{*}\left(\cdot \mid x_{1}\right)$ is obtained by applying Bayes' rule otherwise $\mu^{*}\left(\cdot \mid x_{1}\right)$ is arbitrary.

Any function $\mu^{*}(\cdot \mid \cdot)$ satisfying (iii) or equivalently (iii') will be referred to as a Bayesian posterior belief function. In order to simplify the notation $\Gamma$ we will often simply denote by $\mu$ the probability with which Firm 2 believes that Firm 1 is of the low cost type.

Now $\Gamma$ maximizing $E \Pi_{2}\left(x_{1}, x_{2}, \theta\right)=\mu \Pi_{2}\left(x_{1}, x_{2}, c-\alpha\right)+(1-\mu) \Pi_{2}\left(x_{1}, x_{2}, c\right) \Gamma$ we obtain $\tilde{x}_{2}\left(x_{1}, \mu\right) \Gamma$ the best reply of Firm 2 to the location $x_{1}$ of Firm 1 under the posterior $\mu$ taken here as a parameter:

$$
\tilde{x}_{2}\left(x_{1}, \mu\right)=1 / 3\left(2+x_{1}+\mu \alpha\right) .
$$

Since the best reply is unique given $x_{1}$ and $\mu$ FFirm 2 always uses only pure strategies in equilibrium. Note that $\tilde{x}_{2}(\cdot, \cdot)$ is an increasing function of $x_{1} \Gamma \mu$ Tand $\alpha$.

Let $\hat{\Pi}_{1}\left(x_{1}, \mu, \theta\right)=\Pi_{1}\left(x_{1}, \tilde{x}_{2}\left(x_{1}, \mu\right), \theta\right)$ be the profit function of Firm 1 of type $\theta$ located at $x_{1} \Gamma$ given that Firm 2 chooses its location optimally believing with probability $\mu$ that Firm 1 if of the low cost type. $\hat{\Pi}_{1}\left(x_{1}, \mu, \theta\right)$ is a strictly concave function of $x_{1}$ and an increasing function of $\mu$ for both values of $\theta \Gamma$ as shown in Appendix A. Denoting as $x_{1}(\mu, \theta)$ the optimal location of Firm 1 of type $\theta$ if it is believed by 
Firm 2 to be of the low cost type with probability $\mu$ एwe obtain by maximizing $\hat{\Pi}_{1}\left(x_{1}, \mu, \theta\right)$ in the interval $[0,1 / 2]$ :

$$
x_{1}(\mu, \theta)=(1-\delta(\theta))(1 / 5(2+\mu \alpha))+\delta(\theta) \min \{1 / 5(2+\mu \alpha+3 \alpha), 1 / 2\}
$$

We may rephrase the definition of a PBE in terms of $\Psi_{1}(\cdot) \Gamma \hat{\Pi}_{1}(\cdot) \Gamma$ and $\mu(\cdot \mid \cdot)$ functions. The pair $\left\{\Psi_{1}^{*}(\cdot), \mu^{*}(\cdot \mid \cdot)\right\}$ is a mixed strategy PBE if:

(a) $\forall \theta \in \Theta: \forall x_{1}^{*} \in \operatorname{Supp} \Psi_{1}^{*}(\theta): x_{1}^{*} \in \arg \max _{x_{1} \in X_{1}} \hat{\Pi}_{1}\left(x_{1}, \mu^{*}\left(c-\alpha \mid x_{1}\right), \theta\right)$;

(b) $\mu^{*}(\cdot \mid \cdot)$ is a Bayesian posterior belief function.

Similarly $\left\{x_{1}^{*}(\cdot), \mu^{*}(\cdot \mid \cdot)\right\}$ is a pure strategy PBE if:

$$
\left(\mathrm{a}^{\prime}\right) \forall \theta \in \Theta: x_{1}^{*}(\theta) \in \arg \max _{x_{1} \in X_{1}} \hat{\Pi}_{1}\left(x_{1}, \mu^{*}\left(c-\alpha \mid x_{1}\right), \theta\right) ;
$$

and (b)

It is rather self-evident from the proofs of Propositions $3 \Gamma 4$ Tand 5 that even if the use of mixed strategies with a continuous support were allowed Tin equilibrium only mixed strategies with finite supports would be used. Hence although the set of pure strategies is a continuum in the present model we will limit ourselves to mixed strategies whose support is finiteГand we will denote by $\mathcal{X}_{1 \theta}$ the set of locations over which Firm 1 of type $\theta$ randomizes and by $\nu_{1}\left(x_{1} \mid \theta\right), \theta \in \Theta \Gamma$ the probability with which it chooses $x_{1}$.

As usual in the incomplete information contextГfor a given situation $(\pi, \alpha)$ There exists a continuum of

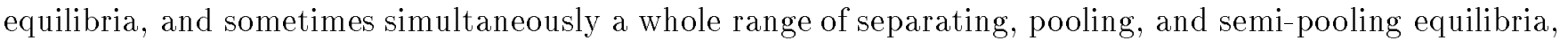
hence the need for a selection device.

\section{THE D1 EQUILIBRIUM REFINEMENT IN MONOTONIC GAMES}

Among the numerous refinements that have been proposed $\Gamma$ the $\mathrm{D} 1$ criterion is quite powerful for a special class of signaling gamesTnamely the monotonic signaling games. For such games one and only one equilibrium is robust to the D1 criterion. In this section Twe rephrase the definition of the D1 refinement in terms of our location modelГand we introduce the notion of the limit posterior belief function that will be used repeatedly in our study of the different kinds of equilibria. Last $\Gamma$ we show that the present game is monotonic.

\subsection{The D1 Equilibrium Refinement}


Let $\mathcal{X}_{1}^{*}=\cup_{\theta \in \Theta} \mathcal{X}_{1 \theta}^{*}$ be the set of equilibrium locations or signals and $\tilde{\mathcal{X}}_{1}=[0,1 / 2] \backslash \mathcal{X}_{1}^{*}$ the set of offthe-equilibrium signals $\Gamma$ the latter locations being chosen with probability 0 in equilibrium. As we saw in the definition of a PBETthe posterior $\mu\left(\theta \mid x_{1}\right)$ is arbitrary for such locations $x_{1} \in \tilde{\mathcal{X}}_{1}$. Refinements serve as reasonable restrictions on $\mu\left(\theta \mid x_{1}\right)$. Criterion D1 is a way to define reasonable beliefs.

Consider a given $\operatorname{PBE} \Gamma\left\{\Psi_{1}^{*}(\cdot), \Psi_{2}^{*}(\cdot), \mu^{*}(\cdot \mid \cdot)\right\} \Gamma$ and let $\Pi_{1}^{*}(\theta)$ be the profit of Firm 1 of type $\theta$ in that equilibrium. Recall that in the case of mixed strategies $\Gamma$ all the locations in $\mathcal{X}_{1 \theta}^{*}$ give the same profits to type $\theta \Gamma$ that is: for $x_{1 \theta}^{*}$ and $x_{1 \theta}^{* \prime}$ in $\mathcal{X}_{1 \theta}^{*} \Gamma \Pi_{1}^{*}(\theta)=\Pi_{1}\left(x_{1 \theta}^{*}, \tilde{x}_{2}\left(x_{1 \theta}^{*}, \mu^{*}\right), \theta\right)=\Pi_{1}\left(x_{1 \theta}^{* \prime}, \tilde{x}_{2}\left(x_{1 \theta}^{* \prime}, \mu^{* \prime}\right), \theta\right) \Gamma$ where $\mu^{*}=\mu^{*}\left(c-\alpha \mid x_{1 \theta}^{*}\right)$ and $\mu^{* \prime}=\mu^{*}\left(c-\alpha \mid x_{1 \theta}^{* \prime}\right)$. Also recall that for any location $x_{1}$ and any posterior belief $\mu$ There is a unique location $\tilde{x}_{2}\left(x_{1}, \mu\right)$ which is the best response to $x_{1}$ Tgiven $\mu$. Let us define $B R\left(x_{1}, \mu\right)$ as $\left\{x_{2} \mid x_{2}=\tilde{x}_{2}\left(x_{1}, \mu\right)\right\}$ and $B R\left(x_{1}\right)$ as $\cup_{\mu \in[0,1]} B R\left(x_{1}, \mu\right)=\left\{x_{2} \mid \exists \mu: x_{2}=\tilde{x}_{2}\left(x_{1}, \mu\right)\right\}$. The set $B R\left(x_{1}, \mu\right)$ contains only one element namely the best response location of Firm 2 to $x_{1}$ given $\mu$. As for $B R\left(x_{1}\right) \Gamma i t$ is the set containing all the "possible" best response locations to $x_{1}$ T that is each location $x_{2}$ which is a best response to $x_{1}$ for some posterior probability $\mu \in[0,1]$. Let us now define:

$$
\begin{aligned}
D\left(\theta \mid x_{1}\right) & =\left\{x_{2} \in B R\left(x_{1}\right) \mid \Pi_{1}^{*}(\theta)<\Pi_{1}\left(x_{1}, x_{2}, \theta\right)\right\}, \\
D^{0}\left(\theta \mid x_{1}\right) & =\left\{x_{2} \in B R\left(x_{1}\right) \mid \Pi_{1}^{*}(\theta)=\Pi_{1}\left(x_{1}, x_{2}, \theta\right)\right\} .
\end{aligned}
$$

The set $D\left(\theta \mid x_{1}\right)$ is the subset of $B R\left(x_{1}\right)$ containing those locations $x_{2}$ which $\Gamma$ if chosen by Firm $2 \Gamma$ would justify a switch by Firm 1 of type $\theta$ from either one of its equilibrium locations to the off-the-equilibrium location $x_{1}$. Its profits are higher at $\left(x_{1}, x_{2}\right) \Gamma x_{2} \in D\left(\theta \mid x_{1}\right) \Gamma$ than at $\left(x_{1 \theta}^{*}, \tilde{x}_{2}\left(x_{1 \theta}^{*}, \mu^{*}\right)\right) \Gamma \mu^{*}=\mu^{*}\left(c-\alpha \mid x_{1 \theta}^{*}\right) \Gamma$ whatever $x_{1 \theta}^{*} \in \mathcal{X}_{1 \theta}^{*}$. Similarly the set $D^{0}\left(\theta \mid x_{1}\right)$ is the set of $x_{2} \in B R\left(x_{1}\right)$ which make a switch to $x_{1}$ a no gain / no loss proposition.

Since $\tilde{x}_{2}\left(x_{1}, \mu\right)$ and therefore $B R\left(x_{1}, \mu\right)$ increase in $\mu \Gamma$ and since $\Pi_{1}\left(x_{1}, x_{2}, \theta\right)$ increases in $x_{2} \Gamma$ we can express the sets $D(\cdot)$ and $D^{0}(\cdot)$ in terms of $\mu$ rather than $x_{2}$ Ta substitution simplifying the use of criterion D1Tas follows:

$$
\begin{aligned}
D\left(\theta \mid x_{1}\right) & =\left\{\mu \mid \Pi_{1}^{*}(\theta)<\hat{\Pi}_{1}\left(x_{1}, \mu, \theta\right)\right\} \\
D^{0}\left(\theta \mid x_{1}\right) & =\left\{\mu \mid \Pi_{1}^{*}(\theta)=\hat{\Pi}_{1}\left(x_{1}, \mu, \theta\right)\right\} .
\end{aligned}
$$

Clearly since $\hat{\Pi}_{1}\left(x_{1}, \mu, \theta\right)$ increases in $\mu \Gamma$ the set $D\left(\theta \mid x_{1}\right) \Gamma$ if nonempty $\Gamma$ will include all the values of $\mu$ above some critical value for which $\Pi_{1}^{*}(\theta)=\hat{\Pi}_{1}\left(x_{1}, \mu, \theta\right)$ Tvalue which is indeed the only element of the set $D^{0}\left(\theta \mid x_{1}\right)$ if nonempty. Before characterizing this critical probability value $\mathrm{let}$ us state criterion D1. Again $\Gamma$ consider a given $\operatorname{PBE} \Gamma\left\{\Psi_{1}^{*}(\cdot), \Psi_{2}^{*}(\cdot), \mu^{*}(\cdot \mid \cdot)\right\}$ Tand an off-the-equilibrium signal $x_{1}$. Then:

$$
\left\{\exists \theta, \theta^{\prime}, \theta \neq \theta^{\prime}: D\left(\theta \mid x_{1}\right) \cup D^{0}\left(\theta \mid x_{1}\right) \subset D\left(\theta^{\prime} \mid x_{1}\right)\right\} \Longrightarrow \mu^{*}\left(\theta \mid x_{1}\right)=0 .
$$


In other words Tif Firm 1 of type $\theta^{\prime}$ has an incentive to deviate when Firm 1 of type $\theta$ has a weak incentive to deviate then Firm 2 should not assign a positive probability that $x_{1}$ has been chosen by Firm 1 of type $\theta$. Phrased differentlyTcriterion D1 stipulates that it is reasonable to suppose with probability 1 that the off-the-equilibrium location $x_{1}$ has been chosen by the type which has the most to gain from it Tin the precise sense of (16).

\subsection{The Limit Posterior Probability Function}

The critical posterior probability value is the key to the characterization of the sets of the different kinds of equilibria and to the application of refinement D1. Let us consider the set of locations of Firm 1 of type $\theta$ not strictly dominated by some given $\left(\dot{x}_{1}, \dot{\mu}\right) \Gamma$ that is $\Gamma$ the set of locations $x_{1}$ such that there exist beliefs $\mu$ with $\hat{\Pi}_{1}\left(x_{1}, \mu, \theta\right) \geq \hat{\Pi}_{1}\left(\dot{x}_{1}, \dot{\mu}, \theta\right)$. As $\hat{\Pi}(\cdot)$ is strictly concave in $x_{1}$ and increasing in $\mu \Gamma$ this set is an interval $\left[x_{\min }\left(\dot{x}_{1}, \dot{\mu}, \theta\right), x_{\max }\left(\dot{x}_{1}, \dot{\mu}, \theta\right)\right]$ where $x_{\min }(\cdot)$ is the smallest root of the second order equation $\hat{\Pi}_{1}\left(x_{1}, 1, \theta\right)=\hat{\Pi}_{1}\left(\dot{x}_{1}, \dot{\mu}, \theta\right) \Gamma$ which is always positive $\Gamma$ and $x_{\max }(\cdot)$ is the largest root if less than $1 / 2$ and equal to $1 / 2$ otherwise.

Consider a location $x_{1} \in\left[x_{\min }\left(\dot{x}_{1}, \dot{\mu}, \theta\right), x_{\max }\left(\dot{x}_{1}, \dot{\mu}, \theta\right)\right]$. Let $\mu\left(x_{1}, \dot{x}_{1}, \dot{\mu}, \theta\right)$ be either the solution of $\hat{\Pi}_{1}\left(x_{1}, \mu, \theta\right)=\hat{\Pi}_{1}\left(\dot{x}_{1}, \dot{\mu}, \theta\right)$ if such a solution exists $\Gamma$ or 0 if not. For example $\Gamma$ suppose $x_{1}(0, \theta)<1 / 2$ and consider $\dot{x}_{1}=x_{1}(0, \theta)+\epsilon<1 / 2 \Gamma \epsilon>0 \Gamma$ and $\dot{\mu}=0$; since $\hat{\Pi}_{1}\left(x_{1}(0, \theta), 0, \theta\right)>\hat{\Pi}_{1}\left(x_{1}(0, \theta)+\epsilon, 0, \theta\right)$ and $\hat{\Pi}(\cdot, \mu, \theta)$ is increasing in $\mu \Gamma$ the equation $\hat{\Pi}_{1}\left(x_{1}(0, \theta), \mu, \theta\right)=\hat{\Pi}_{1}\left(x_{1}(0, \theta)+\epsilon, 0, \theta\right)$ has no solution. As far as $\mu\left(x_{1}, \dot{x}_{1}, \dot{\mu}, \theta\right)>0$ Tit is the highest value of $\mu$ for which Firm 1 of type $\theta$ will prefer to stay at $\dot{x}_{1}$ and face a Firm 2 with posterior $\dot{\mu}$ rather than to switch to $x_{1}$ and face a Firm 2 with posterior $\mu$. For $x_{1} \in\left[0, x_{\min }\left(\dot{x}_{1}, \dot{\mu}, \theta\right)\right) \cup\left(x_{\max }\left(\dot{x}_{1}, \dot{\mu}, \theta\right), 1 / 2\right] \Gamma$ let $\mu\left(x_{1}, \dot{x}_{1}, \dot{\mu}, \theta\right)$ be equal to 1 ; then for any $x_{1} \in[0,1 / 2] \Gamma$ a switch from $\left(\dot{x}_{1}, \dot{\mu}\right)$ to $\left(x_{1}, \mu\right)$ is undesirable for Firm 1 of type $\theta$ if $\mu<\mu\left(x_{1}, \dot{x}_{1}, \dot{\mu}, \theta\right)$.

Note that if $\left(x_{1}^{\prime}, \mu^{\prime}\right)$ and $\left(x_{1}^{\prime \prime}, \mu^{\prime \prime}\right)$ give the same profits to Firm 1 of type $\theta \Gamma$ that is $\Gamma$ if $\hat{\Pi}_{1}\left(x_{1}^{\prime}, \mu^{\prime}, \theta\right)=$ $\hat{\Pi}_{1}\left(x_{1}^{\prime \prime}, \mu^{\prime \prime}, \theta\right) \Gamma$ then for any $x_{1}: \mu\left(x_{1}, x_{1}^{\prime}, \mu^{\prime}, \theta\right)=\mu\left(x_{1}, x_{1}^{\prime \prime}, \mu^{\prime \prime}, \theta\right)$. Since in equilibrium $\hat{\Pi}_{1}\left(x_{1 \theta}^{*}, \mu^{*}, \theta\right)=$ $\hat{\Pi}_{1}\left(x_{1 \theta}^{* \prime}, \mu^{* \prime}, \theta\right)$ whenever $\left\{x_{1 \theta}^{*}, x_{1 \theta}^{* \prime}\right\} \subseteq \mathcal{X}_{1 \theta}^{*} \Gamma \mu^{*}=\mu^{*}\left(c-\alpha \mid x_{1 \theta}^{*}\right)$ and $\mu^{* \prime}=\mu^{*}\left(c-\alpha \mid x_{1 \theta}^{* \prime}\right) \Gamma$ using the limit probability function $\Gamma$ we may redefine criterion $\mathrm{D} 1$ as follows: it is reasonable for Firm 2 Tobserving $x_{1} \Gamma$ to assign probability 0 to type $\theta$ and probability 1 to the other type $\theta^{\prime}$ if $\mu\left(x_{1}, x_{1 \theta}^{*}, \mu^{*}, \theta\right)>\mu\left(x_{1}, x_{1 \theta^{\prime}}^{*}, \mu^{* \prime}, \theta^{\prime}\right) \Gamma$ where $\mu^{*}=\mu^{*}\left(c-\alpha \mid x_{1 \theta}^{*}\right)$ and $\mu^{* \prime}=\mu^{*}\left(c-\alpha \mid x_{1 \theta}^{* \prime}\right)$. Indeed $\Gamma$ we can rewrite (14) and (15) as follows:

$$
\begin{aligned}
\forall \theta \in \Theta, \forall x_{1 \theta}^{*} \in \mathcal{X}_{1 \theta}^{*}: & \\
D\left(\theta \mid x_{1}\right) & =\left\{\mu \mid \mu>\mu\left(x_{1}, x_{1 \theta}^{*}, \mu^{*}, \theta\right)\right\}, \\
D^{0}\left(\theta \mid x_{1}\right) & =\left\{\mu \mid \mu=\mu\left(x_{1}, x_{1 \theta}^{*}, \mu^{*}, \theta\right)\right\} .
\end{aligned}
$$


What makes the use of the limit posterior probability functions an easy and efficient device to determine the locations robust to D1 in the present location model are the following single crossing properties of the $\mu\left(x_{1}, \dot{x}, \dot{\mu}, \theta\right)$ functions.

Proposition 1: Let two pairs of locations and posterior beliefs $\left(x_{1}^{\prime}, \mu^{\prime}\right)$ and $\left(x_{1}^{\prime \prime}, \mu^{\prime \prime}\right)$ be such that:

$$
\begin{aligned}
x_{1}^{\prime} & \in\left[x_{\min }\left(x_{1}(0, c), 0, c\right), x_{\max }\left(x_{1}(0, c), 0, c\right)\right], \\
\mu^{\prime} & \geq \mu\left(x_{1}^{\prime}, x_{1}(0, c), 0, c\right), \\
x^{\prime \prime} & \in\left[x_{\min }\left(x_{1}(0, c-\alpha), 0, c-\alpha\right), x_{\max }\left(x_{1}(0, c-\alpha), 0, c-\alpha\right)\right], \\
\mu^{\prime \prime} & \geq \mu\left(x_{1}^{\prime \prime}, x_{1}(0, c-\alpha), 0, c-\alpha\right) .
\end{aligned}
$$

If there exists a third pair $\left(\dot{x}_{1}, \dot{\mu}\right)$ such that:

$$
\dot{\mu}<1 \text { and } \mu\left(\dot{x}_{1}, x_{1}^{\prime}, \mu^{\prime}, c\right)=\dot{\mu}=\mu\left(\dot{x}_{1}, x_{1}^{\prime \prime}, \mu^{\prime \prime}, c-\alpha\right) \text {, }
$$

then this last pair is unique. $\|$

Proof: See Appendix B.

Proposition 2: Let $\left(\dot{x}_{1}, \dot{\mu}\right)$ be such that:

- either $\dot{\mu}<1$, and $\mu\left(\dot{x}_{1}, x_{1}^{\prime}, \mu^{\prime}, c\right)=\dot{\mu}=\mu\left(\dot{x}_{1}, x_{1}^{\prime \prime}, \mu^{\prime \prime}, c-\alpha\right)$ for some pairs $\left(x_{1}^{\prime}, \mu^{\prime}\right)$ and $\left(x_{1}^{\prime \prime}, \mu^{\prime \prime}\right)$ satisfying respectively (19) $\Gamma(20) \Gamma a n d(21) \Gamma(22)$

- or $\dot{\mu}=1, \dot{x}_{1}=x_{\min }\left(\dot{x}_{1}, \dot{\mu}, \theta\right), \theta \in \Theta \Gamma$ and $\dot{x}_{1} \geq x_{\min }\left(x_{1}(0, c-\alpha), 0, c-\alpha\right)$

- or $\dot{\mu}=1, \dot{x}_{1}=x_{\max }\left(\dot{x}_{1}, \dot{\mu}, \theta\right), \theta \in \Theta \Gamma$ and $\dot{x}_{1} \leq x_{\max }\left(x_{1}(0, c), 0, c\right)$

then:

- for any $x_{1} \in\left(x_{\min }\left(\dot{x}_{1}, \dot{\mu}, c\right), \dot{x}_{1}\right)$ :

$$
\mu\left(x_{1}, \dot{x}_{1}, \dot{\mu}, c\right)<\mu\left(x_{1}, \dot{x}_{1}, \dot{\mu}, c-\alpha\right)
$$

- for any $x_{1} \in\left(\dot{x}_{1}, x_{\max }\left(\dot{x}_{1}, \dot{\mu}, c-\alpha\right)\right)$ :

$$
\mu\left(x_{1}, \dot{x}_{1}, \dot{\mu}, c\right)>\mu\left(x_{1}, \dot{x}_{1}, \dot{\mu}, c-\alpha\right) . \|
$$


Proof: See Appendix B.

The working of propositions 1 and 2 is illustrated on Figure 2 (Appendix C) and Figures 3 and 4 (Appendix E). The fundamental property underlying these propositions is that $\Gamma$ for any given $\left(x_{1}, \dot{x}_{1}, \dot{\mu}\right) \Gamma$ such that $\mu\left(x_{1}, \dot{x}_{1}, \dot{\mu}, \theta\right)<1, \theta \in \Theta \Gamma$ we have:

$$
\frac{\partial \mu\left(x_{1}, \dot{x}_{1}, \dot{\mu}, c\right)}{\partial x_{1}}=-\frac{\partial \hat{\Pi}_{1}\left(x_{1}, \mu, c\right) / \partial x_{1}}{\partial \hat{\Pi}_{1}\left(x_{1}, \mu, c\right) / \partial \mu}>-\frac{\partial \hat{\Pi}_{1}\left(x_{1}, \mu, c-\alpha\right) / \partial x_{1}}{\partial \hat{\Pi}_{1}\left(x_{1}, \mu, c-\alpha\right) / \partial \mu}=\frac{\partial \mu\left(x_{1}, \dot{x}_{1}, \dot{\mu}, c-\alpha\right)}{\partial x_{1}}
$$

In other terms 5 the absolute value of the ratio of marginal profits with respect to location and posterior beliefs (to be identified as a low cost type) is always higher for the high cost type than for the low cost type. Since at $x_{1}=\dot{x}_{1}$ we have $\mu\left(x_{1}, \dot{x}_{1}, \dot{\mu}, c\right)=\dot{\mu}=\mu\left(x_{1}, \dot{x}_{1}, \dot{\mu}, c-\alpha\right) \Gamma$ then the above inequality implies both proposition 1 and proposition 2 .

\subsection{Monotonic Games and the Uniqueness of D1 Equilibrium}

The sequential spatial model we consider in this paper is a monotonic signaling game Tas defined by Cho and Sobel [3]. They show that in such games The equilibrium refinement criteria D1Tuniversal divinity (UD) Tand never a weak best response (NWBR) are all equivalent $\Gamma$ hence lending additional support for using D1 in the present context. Cho and Sobel's definition of a monotonic signaling gameTexpressed in terms of our model $\Gamma$ is as follows: the location game is monotonic if $\forall x_{1} \in[0,1 / 2] \Gamma \forall x_{2}$ and $x_{2}^{\prime} \in B R\left(x_{1}\right) \Gamma$ whenever one type of Firm 1 prefers $\left(x_{1}, x_{2}\right)$ to $\left(x_{1}, x_{2}^{\prime}\right)$ T the other type of Firm 1 does so as well. Since $\partial \Pi_{1}\left(x_{1}, x_{2}, c\right) / \partial x_{2}>0$ and $\partial \Pi_{1}\left(x_{1}, x_{2}, c-\alpha\right) / \partial x_{2}>0 \Gamma$ we have for all locations $x_{2}$ and $x_{2}^{\prime} \in[1 / 2,1] \Gamma$ and a fortiori for those in $B R\left(x_{1}\right)$ :

$$
\left\{\Pi_{1}\left(x_{1}, x_{2}, c\right)>\Pi_{1}\left(x_{1}, x_{2}^{\prime}, c\right)\right\} \quad \text { iff } \quad\left\{\Pi_{1}\left(x_{1}, x_{2}, c-\alpha\right)>\Pi_{1}\left(x_{1}, x_{2}^{\prime}, c-\alpha\right)\right\} .
$$

Each inequality implies $x_{2}>x_{2}^{\prime}$ which Tin turn Timplies the other inequality.

Let us redefine the types of Firm 1 from $\theta \in \Theta=\{c-\alpha, c\}$ to $\tau \in\{0, \alpha\}$ so that the high cost type appears as the lower type which is the one trying to imitate the higher type. This will be useful in [A6] below. Cho and Sobel's sufficient conditions to obtain the existence and uniqueness of a D1-equilibrium are the following: [A1] the set of signals $\Gamma$ that is $\Gamma$ the set of possible locations of Firm $1 \Gamma$ is a compact interval and the set of actions by the receiver $\Gamma$ that is the set of possible locations of Firm $2 \Gamma$ is also a compact interval; [A2] $\Pi_{1}\left(x_{1}, x_{2}, \tau\right)$ is continuous in $\left(x_{1}, x_{2}\right)$ for all $\tau \in\{0, \alpha\}$; [A3] monotonicity as defined above; [A4] $\Pi_{2}\left(x_{1}, x_{2}, \tau\right)$ is a continuous function of $\left(x_{1}, x_{2}\right)$ for all $\tau$ and a strictly quasi-concave differentiable function of $x_{2}$; [A5] $\partial \Pi_{2}\left(x_{1}, x_{2}, \tau\right) / \partial x_{2}$ is a strictly increasing function of $\tau$; [A6] if $\tau<\tau^{\prime}$ and $x_{1}<x_{1}^{\prime} \Gamma$ then $\left\{\Pi_{1}\left(x_{1}, x_{2}, \tau\right) \leq \Pi_{1}\left(x_{1}^{\prime}, x_{2}^{\prime}, \tau\right)\right\} \Rightarrow\left\{\Pi_{1}\left(x_{1}, x_{2}, \tau^{\prime}\right)<\Pi_{1}\left(x_{1}^{\prime}, x_{2}^{\prime}, \tau^{\prime}\right)\right\} ;[\mathrm{A} 7] \forall \tau \Gamma \Pi_{1}\left(x_{1}, x_{2}\left(x_{1}, \tau\right), \tau\right)$ is a strictly quasi-concave function of $x_{1}$. 
It is clear that A1 and A2 hold in the present game and we just showed that A3 is also satisfied. It is straightforward to verify that $\mathrm{A} 4$ holds $\Gamma$ and noting that $\tau$ increases from $0 \Gamma$ for the high cost type $\Gamma$ to $\alpha \Gamma$ for the low cost type $\mathrm{Cit}$ is easy to check that A5 is satisfied too. Condition A6 states that if the high cost Firm 1 makes at least as much profit in situation $\left(x_{1}^{\prime}, x_{2}^{\prime}\right)$ as in situation $\left(x_{1}, x_{2}\right)$ with $x_{1}^{\prime}>x_{1}$ Tthen the low cost Firm 1 does strictly better in $\left(x_{1}^{\prime}, x_{2}^{\prime}\right)$ than in $\left(x_{1}, x_{2}\right)$. This condition is indeed implied by a single crossing property: $-\left(\partial \Pi_{1} / \partial x_{1}\right) /\left(\partial \Pi_{2} / \partial x_{2}\right)$ is decreasing with $\tau$. This expression is equal here to $\left(3 x_{1}-x_{2}-\tau\right) /\left(x_{1}+x_{2}+\tau\right) \Gamma \tau \in\{0, \alpha\}$. Hence $\Gamma$ condition A6 is satisfied. Finally $\Pi_{1}\left(x_{1}, x_{2}\left(x_{1}, \tau\right), \tau\right)$ is strictly concave in $x_{1}$ for both values of $\tau$. Hence $\mathrm{A} 1$ to $\mathrm{A} 7$ are verified. For each situation in the $(\pi, \alpha)$-space $\Gamma$ there exists a unique D1 equilibrium in the present location model.

\section{THE PERFECT BAYESIAN D1 EQUILIBRIA}

For each type of equilibrium $\Gamma$ we describe the set of equilibria and identify those which are robust to D1. This will allow us to draw a map in the $(\pi, \alpha)$-spaceГgiving the unique equilibrium predicted in each possible situation defined by $(\pi, \alpha)$ for $\pi \in(0,1)$ and $\alpha \in(0,1 / 2)$.

\subsection{The Separating Equilibria}

When they exist $\Gamma$ such equilibria are pure strategy equilibria Tthe high cost Firm 1 locating at $x_{1}(0, c)$ and the low cost type locating within $\left[x_{\max }\left(x_{1}(0, c), 0, c\right), x_{\max }\left(x_{1}(0, c-\alpha), 0, c-\alpha\right)\right]$ Tprovided that this interval not be degenerate. This will be the case if the cost discrepancy is not too high Tat most equal to some limit we will denote by $\hat{\alpha}_{c}$. As shown in Appendix CTfor $\alpha<\hat{\alpha}_{c}: x_{\max }\left(x_{1}(0, c), 0, c\right)<x_{\max }\left(x_{1}(0, c-\alpha), 0, c-\alpha\right)$; for $\alpha \geq \hat{\alpha}_{c}: x_{\max }\left(x_{1}(0, c), 0, c\right)=x_{\max }\left(x_{1}(0, c-\alpha), 0, c-\alpha\right)=1 / 2$ and $\mu\left(1 / 2, x_{1}(0, c), 0, c\right) \leq 1 \Gamma$ with the equality if $\alpha=\hat{\alpha}_{c}$ and the strict inequality if $\alpha>\hat{\alpha}_{c}$. The value of $\hat{\alpha}_{c}$ is $9 / \sqrt{5}-4<1 / 2$. For $\alpha<\hat{\alpha}_{c}$ there exists a whole continuum of pure strategy equilibria $\Gamma$ whose measure first increases from 0 at $x=0$ and then decreases and goes back to 0 at $\alpha=\hat{\alpha}_{c}$. For $\alpha=\hat{\alpha}_{c} \Gamma$ there exists a unique separating equilibrium $\Gamma$ and for $\alpha>\hat{\alpha}_{c}$ Tno separating equilibrium exists. When there exists a continuum of equilibria $\Gamma$ the only D1 equilibrium is the least distorting equilibrium Tas measured with respect to the full information equilibrium. More preciselyГwe have:

Proposition 3: All the separating equilibria are pure strategy equilibria. Such equilibria exist if the cost discrepancy is not too high $\Gamma$ namely if $\alpha \in\left[0, \hat{\alpha}_{c}\right] \Gamma$ whatever the priors. If $\alpha \in\left(0, \hat{\alpha}_{c}\right) \Gamma$ there exists a continuum of separating equilibria: $x_{1 c}^{*}=x_{1}(0, c)$ and $x_{1 c-\alpha}^{*} \in\left[x_{\max }\left(x_{1}(0, c), 0, c\right), x_{\max }\left(x_{1}(0, c-\alpha), 0, c-\right.\right.$ 
$\alpha)$ Teach one supported by any posterior beliefs function $\mu^{*}\left(c-\alpha \mid x_{1}\right)$ such that:

$$
\mu^{*}\left(c-\alpha \mid x_{1}\right)\left\{\begin{array}{l}
=0 \quad \text { if } \quad x_{1}=x_{1 c}^{*}, \\
=1 \quad \text { if } \quad x_{1}=x_{1 c-\alpha}^{*}, \\
\leq \min \left\{\mu\left(x_{1}, x_{1 c}^{*}, 0, c\right), \mu\left(x_{1}, x_{1 c-\alpha}^{*}, 1, c-\alpha\right)\right\} \quad \text { otherwise. }
\end{array}\right.
$$

If $\alpha=\hat{\alpha}_{c}$ Tthere exists only one separating equilibrium: $x_{1 c}^{*}=x_{1}(0, c)$ and $x_{1 c-\alpha}^{*}=1 / 2$ supported by the same kind of posterior beliefs. For $\alpha \in\left(0, \hat{\alpha}_{c}\right] \Gamma$ the unique D1 equilibrium among the separating equilibria is the following: $x_{1 c}^{*}=x_{1}(0, c)$ and $x_{1 c-\alpha}^{*}=x_{\max }\left(x_{1}(0, c), 0, c\right) . \|$

Proof: See Appendix C.

Since from (13) $\Gamma x_{\max }\left(x_{1}(0, c), 0, c\right)>x_{1}(1, c-\alpha)$ when $\alpha \leq \hat{\alpha}_{c} \Gamma$ then the $\mathrm{D} 1$ separating equilibria involve a distortion in the location of Firm 1. Although Firm 1 of the high cost type locates at its full information locationГFirm 1 of the low cost type generally locates to the right of its full information location $\Gamma$ closer to the center of the market. But the distortion is the smallest possible given the incomplete information structure compatible with a separating equilibrium.

\subsection{The Pooling Equilibria}

As for the case of separating equilibriaT pooling equilibria are always pure strategy equilibria. Now Twe must distinguish both according to the values of $\alpha$ and the value of the prior belief $\pi$. For any cost advantage $\alpha \Gamma$ there is a whole range of pooling equilibria $\Gamma$ whose measure increases with $\alpha \Gamma$ generally sustained by a continuum of prior beliefs and Bayesian posterior belief functions. However Tthe unique pooling equilibria surviving the application of criterion D1 is the one in which both types of Firm 1 locate at the center of the marketTprovided that the prior probability that Firm 1 is of the low cost type is higher than a strictly positive bound. We have:

Proposition 4: All the pooling equilibria are pure strategy equilibria. For any cost discrepancy $\alpha \in$ $(0,1 / 2)$ Teach location $x_{1}^{*} \in\left(x_{\min }\left(x_{1}(0, c-\alpha), 0, c-\alpha\right), x_{\max }\left(x_{1}(0, c), 0, c\right)\right)$ may be a pooling equilibrium location $\Gamma$ provided that:

- the prior $\pi$ be sufficiently high:

$$
\pi \geq \max \left\{\mu\left(x_{1}^{*}, x_{1}(0, \theta), 0, \theta\right), \theta \in \Theta\right\} ;
$$

- the posterior belief function $\mu^{*}\left(c-\alpha \mid x_{1}\right)$ satisfy:

$$
\mu^{*}\left(c-\alpha \mid x_{1}\right) \begin{cases}=\pi, & \text { if } x=x_{1}^{*} \\ \leq \min \left\{\mu\left(x_{1}, x_{1}^{*}, \pi, \theta\right), \theta \in \Theta\right\}, & \text { otherwise. }\end{cases}
$$


D1 pooling equilibria exist iff $\pi \geq \mu\left(1 / 2, x_{1}(0, c), 0, c\right)$ and $\alpha \in\left(\hat{\alpha}_{c}, 1 / 2\right) \Gamma$ in which case the surviving equilibrium is the center of the market: $x_{1}^{*}=1 / 2 \cdot \|$

Proof: See Appendix D.

The incomplete information pooling equilibrium $\Gamma$ given $\alpha \Gamma$ selected by criterion $\mathrm{D} 1$ is always quite different from the full information equilibrium for the high cost Firm 1 n now locating at the center of the market instead of $x_{1}(0, c)<1 / 2$. For the low cost type $\Gamma$ we must distinguish according to the values of $\alpha$. Remember that under full information $\Gamma$ the low cost type locates at $x_{1}(1, c-\alpha)<1 / 2$ if $\alpha<1 / 8$ and $x_{1}(1, c-\alpha)=1 / 2$ for $\alpha \geq 1 / 8$. Since $\hat{\alpha}_{c}=(9 / \sqrt{5})-4<1 / 8 \Gamma$ the location will be distorted only if $\alpha \in\left(\hat{\alpha}_{c}, 1 / 8\right)$. NoteThoweverTthat even when the location of the low cost type is not distorted $\mathrm{T}$ the doubt subsisting about its true type is prejudicial: Firm 2 will locate nearer the market center than under full information. On the contraryTthe high cost Firm 1 will always be better off at the D1 pooling equilibrium.

\subsection{The Semi-Separating Equilibria}

In all the semi-separating equilibria Teach type of Firm 1 plays at most two different locations and there may be only one location common to the both types 5 so that we have only two kinds of such equilibria: those in which each type $\theta$ randomizes over two locations $\Gamma$ a location $x_{1 \theta}^{*}$ specific to its type and a location

$x_{1}^{*}$ common to both types $\Gamma$ and those in which one of the types $\Gamma$ say $\theta \Gamma$ randomizes over $\left\{x_{1 \theta}^{*}, x_{1}^{*}\right\}$ and the other type $\Gamma \theta^{\prime} \neq \theta \Gamma$ plays $x_{1}^{*} \Gamma$ a pure strategy.

The only kind of semi-separating equilibria existing for all the values of the cost discrepancy $\alpha$ is the one in which only the high cost type randomizesT provided that the prior $\pi$ be sufficiently low. For the other kinds in which either the two types randomize or only the low cost type plays a mixed strategyГthe cost advantage of the low cost type must not be too high Twhatever the prior $\pi$ in the first caseГfor sufficiently high values of $\pi$ only in the second case. In all the equilibria where the high cost type plays a specific location $x_{1 c}^{*}$ Tit is then perfectly identified; hence $\Gamma$ this location must be $x_{1}(0, c) \Gamma$ the location maximizing its profits when clearly perceived as the high cost type. The other locations are less constrained and there generally exists a whole range of possible equilibrium positions.

The equilibrium selected by D1 is for the low cost Firm 1 to locate at the center of the market and for the high cost Firm 1 to randomize over its full information location $x_{1}(0, c)$ and the center of the

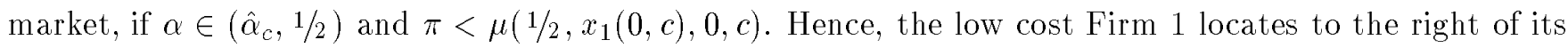
full information location $\Gamma$ while the high cost Firm 1 locates with some probability at its full information location and with the complementary probability at the market center. 
In order to characterize the whole set of semi-separating equilibria Twe introduce the following noteworthy locations. We denote by $\hat{x}_{1}$ the location for which $\mu\left(x_{1}, x_{1}(0, c), 0, c\right)=\mu\left(x_{1}, x_{1}(0, c-\alpha), 0, c-\alpha\right)<1 \Gamma$ that is:

$$
\hat{x}_{1}= \begin{cases}1 / 5\left(\alpha^{2}+6 \alpha+4\right)^{1 / 2}, & \text { if } \alpha \in[0,1 / 6] \\ \left(7920 \alpha^{2}-240 \alpha+1\right)^{1 / 2} / 180 \alpha, & \text { otherwise. }\end{cases}
$$

Clearly $x_{1}(0, c)<\hat{x}_{1}<x_{1}(0, c-\alpha)$. For $\alpha \in\left(0, \hat{\alpha}_{c}\right)$ and $x_{1}^{\prime} \in\left(x_{\max }\left(x_{1}(0, c), 0, c\right), x_{\max }\left(x_{1}(0, c-\alpha), 0, c-\right.\right.$ $\alpha)$ ) we define $\bar{x}_{1}\left(x_{1}^{\prime}\right)$ as the location for which $\mu\left(x_{1}, x_{1}(0, c), 0, c\right)=\mu\left(x_{1}, x_{1}^{\prime}, 1, c-\alpha\right)<1$. This location is also depending on $\alpha$ and $x_{1}(0, c)<\bar{x}_{1}\left(x_{1}^{\prime}\right)<x_{1}(1, c-\alpha)$. Finally let us denote by $\hat{\alpha}_{c-\alpha}$ the upper bound of the values of $\alpha$ for which $x_{\max }\left(x_{1}(0, c-\alpha), 0, c-\alpha\right)<1 / 2: \hat{\alpha}_{c-\alpha}=26-15 \sqrt{3}<\hat{\alpha}_{c}$. For any $\alpha \in\left(\hat{\alpha}_{c-\alpha}, \hat{\alpha}_{c}\right) \Gamma$ we define $\ddot{x}_{1}$ as the location at which $\hat{\Pi}_{1}\left(x_{1}, \mu\left(x_{1}, x,(0, c), 0, c\right), c-\alpha\right)=\hat{\Pi}_{1}(1 / 2,1, c-\alpha) \Gamma$ a location depending on $\alpha$. We have:

Proposition 5: In all the semi-separating equilibria there exists one and only one location chosen in equilibrium by both types of Firm 1 Tand each type chooses two different locations at most.

(i) There exists a continuum of equilibria in which both types of Firm 1 randomize iff $\alpha \in\left(0, \hat{\alpha}_{c}\right] \Gamma$ whatever $\pi$. The equilibrium locations are the following:

$$
\begin{aligned}
& x_{1 c}^{*}=x_{1}(0, c), \\
& x_{1}^{*}\left\{\begin{array}{l}
\in\left[\hat{x}_{1}, x_{\max }\left(x_{1}(0, c), 0, c\right)\right) \text { if } \alpha \in\left(0, \hat{\alpha}_{c-\alpha}\right], \\
\in\left[\ddot{x}_{1}, x_{\max }\left(x_{1}(0, c), 0, c\right)\right) \text { if } \alpha \in\left(\hat{\alpha}_{c-\alpha}, \hat{\alpha}_{c}\right],
\end{array}\right. \\
& x_{1 c-\alpha}^{*}=x_{\max }\left(x_{1}^{*}, \mu^{*}\left(c-\alpha \mid x_{1}^{*}\right), c-\alpha\right) .
\end{aligned}
$$

These equilibrium locations are supported by the posterior beliefs:

$$
\mu^{*}\left(c-\alpha \mid x_{1}\right) \begin{cases}=0, & \text { if } x_{1}=x_{1 c}^{*}, \\ =\mu\left(x_{1}^{*}, x_{1}(0, c), 0, c\right), & \text { if } x_{1}=x_{1}^{*}, \\ =1, & \text { if } x_{1}=x_{1 c-\alpha}^{*}, \\ \leq \min \left\{\mu\left(x_{1}, x_{1}^{*}, \mu^{*}\left(c-\alpha \mid x_{1}^{*}\right), \theta\right), \theta \in \Theta\right\}, & \text { otherwise } \Gamma\end{cases}
$$

and $\Gamma$ given $(\pi, \alpha)$ and $x_{1}^{*} \Gamma$ by a whole range of randomizations $\left\{\nu_{1}\left(x_{1}^{*} \mid c\right), \nu_{1}\left(x_{1}^{*} \mid c-\alpha\right)\right\}$.

(ii) For any $\alpha \in(0,1 / 2) \Gamma$ there exists a continuum of equilibria in which only the high cost Firm 1 randomizes provided that $\pi$ be sufficiently low Tin which case the equilibrium locations are as follows:

$$
\begin{aligned}
& x_{1 c}^{*}=x_{1}(0, c), \\
& x_{1}^{*} \begin{cases}\in\left[\hat{x}_{1}, x_{\max }\left(x_{1}(0, c), 0, c\right)\right), & \text { if } \alpha \in\left(0, \hat{\alpha}_{c}\right], \\
\in\left[\hat{x}_{1}, 1 / 2\right], & \text { if } \alpha \in\left(\hat{\alpha}_{c}, 1 / 2\right),\end{cases}
\end{aligned}
$$


each one supported by:

$$
\mu^{*}\left(c-\alpha \mid x_{1}\right) \begin{cases}=0, & \text { if } x_{1}=x_{1 c}^{*}, \\ =\mu\left(x_{1}^{*}, x_{1}(0, c), 0, c\right), & \text { if } x_{1}=x_{1}^{*}, \\ \leq \min \left\{\mu\left(x_{1}, x_{1}^{*}, \mu^{*}\left(c-\alpha \mid x_{1}^{*}\right), \theta\right), \theta \in \Theta\right\}, & \text { otherwise } \Gamma\end{cases}
$$

and $\Gamma$ given $(\pi, \alpha)$ and $x_{1}^{*}$ Tby only one mixed strategy $\nu_{1}\left(x_{1}^{*} \mid c\right)$ of the high cost type; the condition on $\pi$ is: $\pi<\mu^{*}\left(c-\alpha \mid x_{1}^{*}\right)$.

(iii) There exists a continuum of equilibria in which only the low cost type of Firm 1 randomizes iff $\alpha \in(0,1 / 8)$ Tprovided that $\pi$ be sufficiently high. The equilibrium locations are as follows:

$$
\begin{aligned}
& x_{1 c-\alpha}^{*} \in\left(x_{1}(1, c-\alpha), x_{\max }\left(x_{1}(0, c-\alpha), 0, c-\alpha\right)\right], \\
& x_{1}^{*} \quad\left\{\begin{array}{r}
\in\left(x_{\min }\left(x_{1 c-\alpha}^{*}, 1, c-\alpha\right), x_{1 c-\alpha}^{*}\right), \\
\quad \text { ifffor any } \alpha \in(0,1 / 8), x_{1 c-\alpha}^{*} \in\left(x_{1}(1, c-\alpha), x_{\max }\left(x_{1}(0, c), 0, c\right)\right], \\
\in \quad\left(x_{\min }\left(x_{1 c-\alpha}^{*}, 1, c-\alpha\right), \bar{x}_{1}\left(x_{1 c-\alpha}^{*}\right)\right], \\
\text { if } \alpha \in\left(0, \hat{\alpha}_{c}\right] \text { and } x_{1 c-\alpha}^{*} \in\left(x_{\max }\left(x_{1}(0, c), 0, c\right), x_{\max }\left(x_{1}(0, c-\alpha), 0, c-\alpha\right)\right] .
\end{array}\right.
\end{aligned}
$$

These equilibrium locations are supported by:

$$
\mu^{*}\left(c-\alpha \mid x_{1}^{*}\right) \begin{cases}=1, & \text { if } x_{1}=x_{1 c-\alpha}^{*}, \\ =\mu\left(x_{1}^{*}, x_{1 c-\alpha}^{*}, 1, c-\alpha\right), & \text { if } x_{1}=x_{1}^{*}, \\ \leq \min \left\{\mu\left(x_{1}, x_{1}^{*}, \mu^{*}\left(c-\alpha \mid x_{1}^{*}\right), \theta\right\}, \theta \in \Theta\right\}, & \text { otherwise } \Gamma\end{cases}
$$

and $\Gamma$ given $(\pi, \alpha)$ and $x_{1}^{*}$ Tby only one mixed strategy $\nu_{1}\left(x_{1}^{*} \mid c-\alpha\right)$ of the low cost type; the condition on $\pi$ is: $\pi>\mu\left(x_{1}^{*}, x_{1 c-\alpha}^{*}, 1, c-\alpha\right)$.

(iv) D1 semi-separating equilibria exist if $\pi<\mu\left(1 / 2, x_{1}(0, c), 0, c\right)$ and $\alpha \in\left(\hat{\alpha}_{c}, 1 / 2\right)$. If $(\pi, \alpha)$ is in this region $\Gamma$ then the unique semi-separating equilibrium robust to $\mathrm{D} 1$ is this equilibrium in which only the high cost Firm 1 randomizes over the locations: $x_{1 c}^{*}=x_{1}(0, c)$ and $x_{1}^{*}=1 / 2 \cdot \|$

Proof: See Appendix E.

\subsection{The Mutually Exclusive Domains}

Recapitulating from the previous subsections Twe have that: 
$\star$ for $\alpha \in\left(0, \hat{\alpha}_{c}\right], \pi \in(0,1)$ Tthat is $\Gamma$ for region I on Figure 1 the unique D1 equilibrium is the separating equilibrium which involves the least cost of separation;

$\star$ for $\alpha \in\left(\hat{\alpha}_{c}, 1 / 2\right), \pi \in\left[\mu\left(1 / 2, x_{1}(0, c), 0, c\right), 1\right) \Gamma$ that is Tregion II on Figure 1 The unique D1 equilibrium is the pooling equilibrium at the market center;

$\star$ for $\alpha \in\left(\hat{\alpha}_{c}, 1 / 2\right), \pi \in\left(0, \mu\left(1 / 2, x_{1}(0, c), 0, c\right)\right)$ Tthat isTregion III on Figure 1 the unique D1 equilibrium is the semi-separating equilibrium where only the high cost Firm 1 randomizes over its full information equilibrium and the market centerTand the low cost firm 1 plays a pure strategy at the market center.

Figure 1 about here

What fundamentally happens is that for low cost discrepancies we get the classical result: the two types choose different locations in equilibrium $\Gamma$ the most efficient one incurring a separating cost $\Gamma$ the less efficient one staying at its complete information location. For the efficient type $\mathrm{Tthe}$ separation cost comes from the fact that it must locate nearer the market center than it would have chosen under complete information. As the cost discrepancy increases $\Gamma$ the most efficient type would have to go beyond $1 / 2 \Gamma$ say $1 / 2+\delta$ एwith $\delta>0 \Gamma$ in order to separate from its less efficient twin. All this would work smoothly if Firm 2 were constrained to stay within $(1 / 2+\delta, 1]$. The problem is that Firm 2 is free to choose the most convenient location for itself and switches within $[0,1 / 2+\delta$ ) once Firm 1 goes beyond $1 / 2$. Hence $\Gamma$ the separating power of $1 / 2+\delta$ is not greater than the separating power of $1 / 2-\delta$ and the efficient type stays at $1 / 2$. Doing so creates an incentive for the less efficient type to imitate the more efficient one. But imitation may really succeed only if the prior probability $\pi$ that Firm 1 is of the low cost type is sufficiently high depends on this probability for a given location of Firm 1. If this probability is too low Tthe pooling process is blurred: the high cost Firm 1 will imitate its low cost twin only with a probability less than 1 Thence generating a mixed strategy equilibrium.

\section{CONCLUSION}

This paper has made use of Cho and Sobel [3] to exhibit the outcome of spatial competition $\Gamma$ as predicted by the recent developments of the theory of incomplete information games. It provides an exhaustive description and prediction of the variety of distortions in locations which can arise because of incomplete information. Clearly incomplete information appears to have a major influence on location choices. Although the less efficient type of Firm 1 chooses its complete information location in separating and $\mathrm{Twith}$

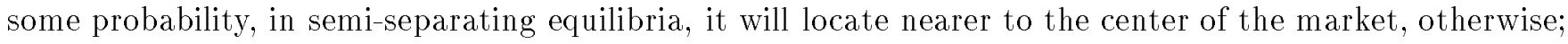
as for the most efficient typeTfor all the values of the cost advantage for which the full information location is not at the market center $\mathrm{T}$ the incomplete information location is nearer to the market center than the 
complete information one. Hence the incomplete information always results in a more aggressive price competition.

From the point of view of signaling models Tour model provides an economically meaningful example of the usefulness of the D1 refinement to narrow down successfully and dramatically the set of equilibria. There are not so many examples of such applications. 


\section{APPENDIX A: PROPERTIES OF THE $\hat{\Pi}_{1}\left(x_{1}, \mu, \theta\right)$ FUNCTIONS}

Substituting $\tilde{x}_{2}\left(x_{1}, \mu\right)=1 / 3\left(x_{1}+\mu x+2\right)$ for $x_{2}$ in the Firm 1's profit function $(2): \Pi_{1}\left(x_{1}, x_{2}, \theta\right)=$ $1 / 4\left(x_{2}-x_{1}\right)\left(3 x_{1}+x_{2}\right)+\delta(\theta)\left[1 / 2 \alpha\left(x_{1}+x_{2}\right)+1 / 4 \alpha^{2}\right] \Gamma$ where $\delta(\theta)=0$ if $\theta=c$ and $\delta(\theta)=1$ if $\theta=c-\alpha \Gamma$ we get:

$$
\hat{\Pi}_{1}\left(x_{1}, \mu, \theta\right)=1 / 36\left[-20 x_{1}^{2}+8(2+\mu \alpha+3 \delta(\theta) \alpha) x_{1}+(2+\mu \alpha+3 \delta(\theta) \alpha)^{2}\right]
$$

with

$$
\begin{aligned}
\frac{\partial \hat{\Pi}_{1}}{\partial \mu} & =1 / 36\left[8 \alpha x_{1}+2 \alpha(2+\mu \alpha+3 \delta(\theta) \alpha)\right]>0, \frac{\partial^{2} \hat{\Pi}_{1}}{\partial \mu^{2}}=1 / 18 \alpha^{2} \\
\frac{\partial \hat{\Pi}_{1}}{\partial x_{1}} & =1 / 9\left[-10 x_{1}+2(2+\mu \alpha+3 \delta(\theta) \alpha)\right], \quad \frac{\partial^{2} \hat{\Pi}_{1}}{\partial x_{1}^{2}}=-10 / 9<0 \\
\frac{\partial^{2} \hat{\Pi}_{1}}{\partial x_{1} \partial \mu} & =2 / 9 x_{1}>0 .
\end{aligned}
$$

The $\hat{\Pi}_{1}($.$) functions are strictly concave in x_{1}$ so that the location $x_{1}(\mu, \theta)$ maximizing $\hat{\Pi}_{1}\left(x_{1}, \mu, \theta\right)$ is unique and equal to:

$$
x_{1}(\mu, \theta)=(1-\delta(\theta))[1 / 5(2+\mu \alpha)]+\delta(\theta) \min \{1 / 5(2+\mu \alpha+3 \alpha), 1 / 2\} .
$$

Note that for a given $\alpha \Gamma$ the two intervals $\left[x_{1}(0, \theta), x_{1}(1, \theta)\right], \theta \in \Theta \Gamma$ do not intersect. Substituting $x_{1}(\mu, \theta)$ for $x_{1}$ in $\hat{\Pi}_{1}\left(x_{1}, \mu, \theta\right)$ Гwe get:

$$
\hat{\Pi}_{1}\left(x_{1}(\mu, \theta), \mu, \theta\right)=\min \left\{5 / 4 x_{1}(\mu, \theta)^{2}, 1 / 36\left[3+4(\mu+3) \alpha+(2+\mu \alpha+3 \alpha)^{2}\right]\right\} .
$$

Hence $\Gamma$ since the two intervals $\left[x_{1}(0, \theta), x_{1}(1, \theta)\right], \theta \in \Theta \Gamma$ do not intersect:

$$
\hat{\Pi}_{1}\left(x_{1}(1, c), 1, c\right)<\hat{\Pi}_{1}\left(x_{1}(0, c-\alpha), 0, c-\alpha\right), \alpha \in(0,1 / 2) .
$$

\section{APPENDIX B: THE LIMIT POSTERIOR PROBABILITY FUNCTION}

In this appendix $\Gamma$ we give all the relevant characteristics of the limit probability functions $\mu\left(x_{1}, \dot{x}_{1}, \dot{\mu}, \theta\right), \theta \in \Theta$. 
(B.1) For the high cost Firm 1 correctly identified as such and located at its profit maximizing location $\Gamma$ that is $\Gamma$ for $\theta=c, \dot{\mu}=0, \dot{x}_{1}=x_{1}(0, c)(=2 / 5<1 / 2)$ Twe have:

$$
\begin{array}{ll}
x_{\min }\left(x_{1}(0, c), 0, c\right)=1 / 5\left(2+\alpha-\Delta^{1 / 2}\right), & \\
x_{\max }\left(x_{1}(0, c), 0, c\right)=\left\{\begin{array}{lll}
1 / 5\left(2+\alpha+\Delta^{1 / 2}\right), & \text { if } & \alpha \leq \hat{\alpha}_{c}=(9 / \sqrt{5})-4, \\
1 / 2, & \text { otherwise } \Gamma
\end{array}\right.
\end{array}
$$

where $\Delta=9\left(1 / 4 \alpha^{2}+\alpha\right)$. The limit probability function $\mu\left(x_{1}, x_{1}(0, c), 0, c\right)$ is continuous and equal to 1 on $\left[0, x_{\min }\left(x_{1}(0, c), 0, c\right)\right] \Gamma$ decreasing from 1 to 0 on $\left(x_{\min }\left(x_{1}(0, c), 0, c\right), x_{1}(0, c)\right] \Gamma$ increasing from 0 to 1 on $\left(x_{1}(0, c), x_{\max }\left(x_{1}(0, c), 0, c\right)\right]$ if the upper bound of this interval is lower than $1 / 2 \Gamma$ and remaining equal to 1 on $\left(x_{\max }\left(x_{1}(0, c), 0, c\right), 1 / 2\right]$ Tincreasing from 0 to a value less than 1 on $\left(x_{1}(0, c), x_{\max }\left(x_{1}(0, c), 0, c\right)\right]$ if $x_{\max }\left(x_{1}(0, c), 0, c\right)=1 / 2$.

(B.2) For the low cost Firm 1 wrongly identified as a high cost one and optimally located given this misperception $\Gamma$ that is $\Gamma$ for $\theta=c-\alpha, \dot{\mu}=0 \Gamma \dot{x}_{1}=x_{1}(0, c-\alpha)$ (either equal to $1 / 5(2+3 \alpha)$ if $\alpha \leq 1 / 6$ or to $1 / 2$ if $\alpha>1 / 6$ ) we have:

$$
\begin{aligned}
& x_{\text {min }}\left(x_{1}(0, c-\alpha), 0, c-\alpha\right)= \begin{cases}1 / 5\left(2+4 \alpha-\Omega^{1 / 2}\right), & \text { if } \alpha \leq 1 / 6, \\
1 / 10\left(4+8 \alpha-\Gamma^{1 / 2}\right), & \text { otherwise } \Gamma\end{cases} \\
& x_{\text {max }}\left(x_{1}(0, c-\alpha), 0, c-\alpha\right)= \begin{cases}1 / 5\left(2+4 \alpha-\Omega^{1 / 2}\right), & \text { if } \alpha \leq \hat{\alpha}_{c-\alpha}=26-15 \sqrt{3}, \\
1 / 2, & \text { otherwise } \Gamma\end{cases}
\end{aligned}
$$

where $\Omega=9\left(\tau / 4 \alpha^{2}+\alpha\right)$ and $\Gamma=99 \alpha^{2}+24 \alpha+1$. The limit probability function $\mu\left(x_{1}, x_{1}(0, c-\right.$ $\alpha), 0, c-\alpha)$ is continuous and equal to 1 on $\left[0, x_{\min }\left(x_{1}(0, c-\alpha), 0, c-\alpha\right)\right] \Gamma$ decreasing from 1 to 0 on $\left(x_{\min }\left(x_{1}(0, c-\alpha), 0, c-\alpha\right), x_{1}(0, c-\alpha)\right]$ Tincreasing from 0 to 1 on $\left(x_{1}(0, c-\alpha), x_{\max }\left(x_{1}(0, c-\alpha), 0, c-\right.\right.$ $\alpha)]$ if $x_{1}(0, c-\alpha)<x_{\max }\left(x_{1}(0, c-\alpha), 0, c-\alpha\right)<1 / 2$ (note that the second inequality implies the first) and remaining equal to 1 on $\left(x_{\max }\left(x_{1}(0, c-\alpha), 0, c-\alpha\right), 1 / 2\right]$ Tincreasing from 0 to a value less than 1 on $\left(x_{1}(0, c-\alpha), x_{\max }\left(x_{1}(0, c-\alpha), 0, c-\alpha\right)\right]$ if $x_{1}(0, c-\alpha)<x_{\max }\left(x_{1}(0, c-\alpha), 0, c-\alpha\right)=1 / 2$.

(B.3) There are important relationships between the limit posterior probability functions $\mu\left(x_{1}, \dot{x}_{1}, \dot{\mu}, c\right)$ and $\mu\left(x_{1}, \dot{x}_{1}, \dot{\mu}, c-\alpha\right)$ Tdefined for a given $\left(\dot{x}_{1}, \dot{\mu}\right)$. By definition:

- the two functions have the same value $\dot{\mu}$ at $x_{1}=\dot{x}_{1}$ :

$$
\mu\left(\dot{x}_{1}, \dot{x}_{1}, \dot{\mu}, c\right)=\mu\left(\dot{x}_{1}, \dot{x}_{1}, \dot{\mu}, c-\alpha\right)=\dot{\mu}
$$

- each function assumes a value less than 1 within the interval $\left(x_{\min }\left(\dot{x}_{1}, \dot{\mu}, \theta\right), x_{\max }\left(\dot{x}_{1}, \dot{\mu}, \theta\right)\right)$ :

$$
x_{1} \in\left(x_{\min }\left(\dot{x}_{1}, \dot{\mu}, \theta\right), x_{\max }\left(\dot{x}_{1}, \dot{\mu}, \theta\right)\right) \Rightarrow \mu\left(x_{1}, \dot{x}_{1}, \theta\right)<1
$$


- defining $A_{\theta}$ as the following interval:

$$
A_{\theta}= \begin{cases}\left(x_{\min }\left(x_{1}(0, \theta), 0, \theta\right), x_{\max }\left(x_{1}(0, \theta), 0, \theta\right)\right), & \text { if either } \alpha \leq \hat{\alpha}_{c} \text { and } \theta=c \\ & \text { or } \alpha \leq \hat{\alpha}_{c-\alpha} \text { and } \theta=c-\alpha, \\ \left(x_{\min }\left(x_{1}(0, \theta), 0, \theta\right), x_{\max }\left(x_{1}(0, \theta), 0, \theta\right)\right], & \text { otherwise } \Gamma\end{cases}
$$

then:

$$
\begin{aligned}
& x_{1} \in A_{\theta} \quad \Rightarrow \quad \mu\left(x_{1}, x_{1}(0, \theta), 0, \theta\right)<1, \\
& x_{1} \notin A_{\theta} \quad \Rightarrow \quad \mu\left(x_{1}, x_{1}(0, \theta), 0, \theta\right)=1 .
\end{aligned}
$$

(B.4) Proofs of Propositions 1 and 2. Let $\left(x_{1}^{\prime}, \mu^{\prime}\right) \Gamma\left(x_{1}^{\prime \prime}, \mu^{\prime \prime}\right)$ and $\left(\dot{x}_{1}, \dot{\mu}\right)$ be such that:

$$
\begin{aligned}
x_{1}^{\prime} & \in\left[x_{\min }\left(x_{1}(0, c), 0, c\right), x_{\max }\left(x_{1}(0, c), 0, c\right)\right], \mu^{\prime} \geq \mu\left(x_{1}^{\prime}, x_{1}(0, c), 0, c\right), \\
x_{1}^{\prime \prime} & \in\left[x_{\min }\left(x_{1}(0, c-\alpha), 0, c-\alpha\right), x_{\max }\left(x_{1}(0, c-\alpha), 0, c-\alpha\right)\right], \mu^{\prime \prime} \geq \mu\left(x_{1}^{\prime \prime}, x_{1}(0, c-\alpha), 0, c-\alpha\right), \\
\dot{\mu} & <1 \text { and } \mu\left(\dot{x}_{1}, x_{1}^{\prime}, \mu^{\prime}, c\right)=\dot{\mu}=\mu\left(\dot{x}_{1}, x_{1}^{\prime \prime}, \mu^{\prime \prime}, c-\alpha\right) .
\end{aligned}
$$

Note that: $\mu\left(x_{1}, x_{1}^{\prime}, \mu^{\prime}, c\right)=\mu\left(x_{1}, \dot{x}_{1}, \dot{\mu}, c\right)$ and $\mu\left(x_{1}, x_{1}^{\prime \prime}, \mu^{\prime \prime}, c-\alpha\right)=\mu\left(x_{1}, \dot{x}_{1}, \dot{\mu}, c-\alpha\right)$.

For any $x_{1} \in\left[x_{\min }\left(\dot{x}_{1}, \dot{\mu}, \theta\right), x_{\max }\left(\dot{x}_{1}, \dot{\mu}, \theta\right)\right], \theta \in \Theta$ let $\mu\left(x_{1}, \dot{x}_{1}, \dot{\mu}, \theta\right)$ be the relevant root of:

$$
\hat{\Pi}_{1}\left(x_{1}, \mu, \theta\right)=\hat{\Pi}_{1}\left(\dot{x}_{1}, \dot{\mu}, \theta\right)
$$

Differentiating this equation we get:

$$
\frac{\partial \mu\left(x_{1}, \dot{x}_{1}, \dot{\mu}, \theta\right)}{\partial x_{1}}=-\frac{\partial \hat{\Pi}_{1}\left(x_{1}, \mu, \theta\right) / \partial x_{1}}{\partial \hat{\Pi}_{1}\left(x_{1}, \mu, \theta\right) / \partial \mu}
$$

that is $\Gamma$ from (A.2) and (A.3):

$$
\begin{aligned}
\frac{\partial \mu\left(x_{1}, \dot{x}_{1}, \dot{\mu}, c\right)}{\partial x_{1}} & =\frac{-40 x_{1}+8(2+\mu \alpha)}{8 \alpha x_{1}+2 \alpha(2+\mu \alpha)} \\
\frac{\partial \mu\left(x_{1}, \dot{x}_{1}, \dot{\mu}, c-\alpha\right)}{\partial x_{1}} & =\frac{-40 x_{1}+8(2+\mu \alpha+3 \alpha)}{8 \alpha x_{1}+2 \alpha(2+\mu \alpha+3 \alpha)} .
\end{aligned}
$$

Hence:

$$
\frac{\partial \mu\left(x_{1}, \dot{x}_{1}, \dot{\mu}, c\right)}{\partial x_{1}}=\frac{\partial \mu\left(x_{1}, \dot{x}_{1}, \dot{\mu}, c-\alpha\right)}{\partial x_{1}}+\frac{2^{2} 3^{3} x_{1}}{\left(4 x_{1}+2+\mu \alpha\right)\left(4 x_{1}+2+\mu \alpha+3 \alpha\right)}
$$

Since

$$
\frac{\partial \mu\left(x_{1}, \dot{x}_{1}, \dot{\mu}, c\right)}{\partial x_{1}}>\frac{\partial \mu\left(x_{1}, \dot{x}_{1}, \dot{\mu}, c-\alpha\right)}{\partial x_{1}}
$$


then $\left(\dot{x}_{1}, \dot{\mu}\right)$ is the unique pair satisfying the two conditions $\dot{\mu}<1$ and $\mu\left(x_{1}, \dot{x}_{1}, \dot{\mu}, c\right)=\dot{\mu}=\mu\left(x_{1}, \dot{x}_{1}, \dot{\mu}, c-\alpha\right)$ for $x=\dot{x}_{1}$. (B.10) and the continuity of the functions $\mu\left(x_{1}, \ldots, \theta\right), \theta \in \Theta$ Гimply Proposition 2.

\section{APPENDIX C: PROOF OF PROPOSITION 3}

The logic underlying the construction of a separating equilibrium is illustrated on Figure $2 \Gamma$ where the profit functions $\hat{\Pi}_{1}\left(x_{1}, \mu, \theta\right)$ are represented as functions of $x_{1}$.

Figure 2 about here

The curves (1) $\Gamma(2) \Gamma$ and (3) correspond respectively to $\hat{\Pi}_{1}\left(x_{1}, 1, c-\alpha\right) \Gamma$ to $\hat{\Pi}_{1}\left(x_{1}, \mu, c-\alpha\right)$ for $0<\mu<1 \Gamma$ and to $\hat{\Pi}_{1}\left(x_{1}, 0, c-\alpha\right) \Gamma$ that is $\Gamma$ respectively $\mathrm{C}$ to the profits of the low cost Firm 1 when rightly identified by Firm $2(\mu=1)$ Twhen some doubt subsists about its type $(0<\mu<1)$ and when wrongly identified as the high cost type $(\mu=0)$. Curves $\left(1^{\prime}\right) \Gamma\left(2^{\prime}\right) \Gamma$ and $\left(3^{\prime}\right)$ correspond respectively to $\hat{\Pi}_{1}\left(x_{1}, 1, c\right) \Gamma \hat{\Pi}_{1}\left(x_{1}, \mu, c\right)$ for $0<\mu<1 \Gamma$ and to $\hat{\Pi}_{1}\left(x_{1}, 0, c\right)$ Tthat is $\Gamma$ respectively $\Gamma$ to the profits of the high cost type Firm 1 when wrongly identified as the low cost type $\Gamma$ when not clearly distinguished from its low cost twin and when rightly identified as of the high cost type. Curve (4) is the locus of maxima of the functions $\hat{\Pi}_{1}\left(x_{1}, \mu, \theta\right) \Gamma$ $\theta \in \Theta$. Remember that in a separating equilibrium the two types of Firm 1 choose different locations $\Gamma$ that is

$\mathcal{X}_{1 \theta}^{*} \cap \mathcal{X}_{1 \theta^{\prime}}^{*}=\emptyset \Gamma \theta \neq \theta^{\prime}$. Hence $\forall \theta \in \Theta \Gamma \forall x_{1 \theta}^{*} \in \mathcal{X}_{1 \theta}^{*}: \mu^{*}\left(c-\alpha \mid x_{1 \theta}^{*}\right)=\delta(\theta)$.

Consider first the high cost type and suppose that $x_{1} \neq x_{1}(0, c)$ and $x_{1} \in \mathcal{X}_{1 c}^{*}$. Trivially $\hat{\Pi}_{1}\left(x_{1}, 0, c\right)<$ $\hat{\Pi}_{1}\left(x_{1}(0, c), 0, c\right)<\hat{\Pi}_{1}\left(x_{1}(0, c), \mu, c\right)$ if $\mu>0$. Since at $x_{1} \in \mathcal{X}_{1 c}^{*} \Gamma$ Firm 1 of the high cost type must be rightly identified as such $\Gamma$ the above inequality implies that deviating from $x_{1}$ to $x_{1}(0, c)$ would be profitable $\Gamma$ whatever the posteriors of Firm 2 observing the deviation. Hence The only possible equilibrium location of the high cost type of Firm 1 is $x_{1}(0, c)$ Twhich implies that for this type mixed strategies are forbidden. In order that $x_{1}(0, c)$ be the equilibrium location of the high cost type $\Gamma$ the posteriors induced by any off-the-equilibrium location $x_{1} \neq x_{1}(0, c) \Gamma \mu^{*}\left(c-\alpha \mid x_{1}\right) \Gamma$ must be at most equal to $\mu\left(x_{1}, x_{1}(0, c), 0, c\right) \Gamma$ that is Tstrictly less than 1 on the interval $A_{c}$ (see (B.7) and (B.8)).

Consider the low cost type. Its equilibrium location may not be within $\left[0, x_{\min }\left(x_{1}(0, c-\alpha), 0, c-\alpha\right)\right) \cup$ $\left(x_{\max }\left(x_{1}(0, c-\alpha), 0, c-\alpha\right), 1 / 2\right] \Gamma$ since in this interval $\hat{\Pi}_{1}\left(x_{1}, 1, c-\alpha\right)<\hat{\Pi}_{1}\left(x_{1}(0, c-\alpha), 0, c-\alpha\right)<\hat{\Pi}_{1}\left(x_{1}(0, c-\right.$ $\alpha), \mu, c-\alpha), 0<\mu$. A deviation from any given $x_{1}$ in the above interval to $x_{1}(0, c-\alpha)$ would be profitable $\Gamma$ whatever the posteriors of Firm 2 observing the deviation. Note also that its equilibrium location may not be within the interval $A_{c}$ Tsince for any alleged equilibrium location $x_{1}$ in this intervalГwe would have: (1) $\mu^{*}\left(c-\alpha \mid x_{1}\right)<1$ (in order that the high cost type stays at $x_{1}(0, c)$ ) and $(2) \mu^{*}\left(c-\alpha \mid x_{1}\right)=1$ (in order that 
the low cost type be rightly identified as such). Because $x_{\min }\left(x_{1}(0, c), 0, c\right)<x_{\min }\left(x_{1}(0, c-\alpha), 0, c-\alpha\right)$ and $x_{\max }\left(x_{1}(0, c), 0, c\right) \leq x_{\max }\left(x_{1}(0, c-\alpha), 0, c-\alpha\right) \Gamma$ the whole interval $\left[0, x_{\max }\left(x_{1}(0, c), 0, c\right)\right)$ is excluded. Hence $\Gamma$ we are left with the sole interval $\left[x_{\max }\left(x_{1}(0, c), 0, c\right), x_{\max }\left(x_{1}(0, c-\alpha), 0, c-\alpha\right)\right]$. This interval is nonemptyГ provided that $\mu\left(x_{\max }\left(x_{1}(0, c), 0, c\right), x_{1}(0, c), 0, c\right)=1 \Gamma$ which is the case if $\alpha \leq \hat{\alpha}_{c}$. In this case $\hat{\Pi}_{1}\left(x_{1}, 1, c-\alpha\right)$ is decreasing on this interval so that the low cost type may not randomize over several locations That is to say last interval may be the equilibrium location of the low cost typeTprovided that for any off-the-equilibrium location $x_{1}, \mu^{*}\left(c-\alpha \mid x_{1}\right) \leq \mu\left(x_{1}, x_{1 c-\alpha}^{*}, 1, c-\alpha\right)$.

Let us now turn to the working of D1. We first show that any equilibrium $x_{1 c-\alpha}^{*}>x_{\max }\left(x_{1}(0, c-\alpha), 0, c-\right.$ $\alpha)$ is destroyed by D1. For such a location $\Gamma$ consider any alternative location $x_{1}^{*} \in\left(x_{\max }\left(x_{1}(0, c), 0, c\right)\right.$, $\left.x_{1 c-\alpha}^{*}\right)$. First $\Gamma$ we have $\mu\left(x_{1}^{\prime}, x_{1}(0, c), 0, c\right)=\mu\left(x_{1}^{\prime}, x_{1 c}^{*}, 0, c\right)=1$. Second $\Gamma$ since $\hat{\Pi}_{1}\left(x_{1}, 1, c-\alpha\right)$ is decreasing on $\left(x_{\max }\left(x_{1}(0, c), 0, c\right), x_{1 c-\alpha}^{*}\right) \Gamma$ then $\mu\left(x_{1}^{\prime}, x_{1 c-\alpha}^{*}, 1, c-\alpha\right)<1$. Therefore ${ }^{*}$ observing $x_{1}^{\prime} \Gamma$ Firm 2 should conclude $\Gamma$ according to D1 $\Gamma$ that it is facing the low cost type. But $\hat{\Pi}_{1}\left(x_{1}^{\prime}, 1, c-\alpha\right)>\hat{\Pi}_{1}\left(x_{1 c-\alpha}^{*}, 1, c-\alpha\right)=$ $\Pi_{1}^{*}(c-\alpha)$ implies that the deviation is profitable for the low cost type. Let us now show that $x_{1 c-\alpha}^{*}=$ $x_{\max }\left(x_{1}(0, c), 0, c\right)$ is robust to $\mathrm{D} 1$. For this equilibrium location $\Gamma$ the above kind of deviation is no more allowed. Consider first deviations either in $\left[0, x_{\min }\left(x_{1}(0, c), 0, c\right)\right]$ or in $\left(x_{1 c-\alpha}^{*}, 1 / 2\right]$ : no type would gain $\Gamma$ whatever the posterior beliefs of Firm 2. Consider now deviations $x_{1}^{\prime}$ within $\left(x_{\min }\left(x_{1}(0, c), 0, c\right), x_{1 c-\alpha}^{*}\right)$ : from Proposition 2 (substitute $x_{1 c-\alpha}^{*}=x_{\max }\left(x_{1}(0, c), 0, c\right)$ for $\dot{x}_{1}$ in $(25)$ Tnote that $\mu\left(x_{1 c-\alpha}^{*}, x,(0, c), 0, c\right)=$ $\mu^{*}\left(c-\alpha \mid x_{1 c-\alpha}^{*}\right)=1=\dot{\mu}$ and apply (26)) $\Gamma$ we get $\mu\left(x_{1}^{\prime}, x_{1 c}^{*}, 0, c\right)<\mu\left(x_{1}^{\prime}, x_{1 c-\alpha}^{*}, 1, c-\alpha\right) \Gamma$ so that $\Gamma$ according to D1ГFirm 2 should conclude that it is facing the high cost Firm 1 . Since $\hat{\Pi}_{1}\left(x_{1 c-\alpha}^{*}, 1, c-\alpha\right)>$ $\hat{\Pi}_{1}\left(x_{1 c-\alpha}^{*}, \mu\left(x_{1 c-\alpha}^{*}, x_{1}(0, c-\alpha), 0, c-\alpha\right), c-\alpha\right)=\hat{\Pi}_{1}\left(x_{1}(0, c-\alpha), 0, c-\alpha\right) \geq \hat{\Pi}_{1}\left(x_{1}^{\prime}, 0, c-\alpha\right)$ Tsuch a deviation would imply a loss for the low cost type. As for the high cost type we have $\hat{\Pi}_{1}\left(x_{1}^{\prime}, 0, c\right) \leq \hat{\Pi}_{1}\left(x_{1}(0, c), 0, c\right) \Gamma$ with the strict inequality if $x_{1}^{\prime} \neq x_{1}(0, c)$ rso that the deviation is worthless.

\section{APPENDIX D: PROOF OF PROPOSITION 4}

In a pooling equilibrium $\Gamma$ the equilibrium locations chosen by the two types of Firm 1 are the sameI $\mathcal{X}_{1 \theta}^{*}=\mathcal{X}_{1}^{*}, \theta \in \Theta \Gamma$ and if mixed strategies are used $\Gamma$ the probabilities with which the two types randomize over $\mathcal{X}_{1}^{*}$ are the same so that observing any $x_{1}^{*} \in \mathcal{X}_{1}^{*} \Gamma$ the posterior beliefs of Firm 2 must be equal to the priors: $\mu^{*}\left(c-\alpha \mid x_{1}^{*}\right)=\pi$. Thus $\Gamma$ the equilibrium profits of the high and low cost types amount respectively to $\hat{\Pi}_{1}\left(x_{1}^{*}, \pi, c\right)$ and $\hat{\Pi}_{1}\left(x_{1}^{*}, \pi, c-\alpha\right)$.

For each type $\theta \Gamma$ the equilibrium profits must be at least equal to $\hat{\Pi}_{1}\left(x_{1}(0, \theta), 0, \theta\right)$ that type $\theta$ would obtain by choosing the location maximizing its profit when perceived Tright or wrong Tas the high cost type. 
If not Tthe type $\theta$ in question would be sure to obtain higher profits by deviating from the alleged equilibrium to $x_{1}(0, \theta)$ (the argument is the same as for separating equilibria $\Gamma$ but as we shall see $\Gamma$ the consequences differ because we are looking for a different kind of equilibrium). Hence for any prior $\pi \in(0,1)$, any equilibrium location must be within the following interval $A$ :

$$
A=\bigcap_{\theta \in \Theta} A_{\theta}
$$

that is:

$$
A= \begin{cases}\left(x_{\min }\left(x_{1}(0, c-\alpha), 0, c-\alpha\right), x_{\max }\left(x_{1}(0, c), 0, c\right)\right), & \text { if } \alpha \leq \hat{\alpha}_{c} \\ \left(x_{\min }\left(x_{1}(0, c-\alpha), 0, c-\alpha\right), 1 / 2\right], & \text { otherwise }\end{cases}
$$

In order that a location $x_{1}^{*} \in A$ be an equilibrium location $\Gamma$ it is necessary that $\pi$ be at least equal to $\max \left\{\mu\left(x_{1}^{*}, x_{1}(0, \theta), 0, \theta\right), \theta \in \Theta\right\}$ and that for any off-the-equilibrium location $x_{1}^{\prime} \Gamma$ the posterior beliefs $\mu^{*}\left(c-\alpha \mid x_{1}^{\prime}\right)$ not be too high $\Gamma$ namely $\mu^{*}\left(c-\alpha \mid x_{1}^{\prime}\right) \leq \min \left\{\mu\left(x_{1}^{\prime}, x_{1}^{*}, \pi, \theta\right), \theta \in \Theta\right\}$. If either one of these conditions were to fail for some type $\theta \Gamma$ this type would deviate profitably from $x_{1}^{*}$ to either $x_{1}(0, \theta)$ or $x_{1}^{\prime} \Gamma$ according to whether it is the first or the second condition which is not met. It is important to note that $\max \left\{\mu\left(x_{1}, x_{1}(0, \theta), 0, \theta\right), \theta \in \Theta\right\}$ is strictly positive for any $x_{1} \in A$ Tso that the lower bound of the range of values of $\pi$ for which pooling equilibria exist $\Gamma$ is strictly positive. On the other hand $\Gamma$ there is no restriction on the range of values of the cost discrepancy $\alpha$. If the play is in pure strategies $\Gamma$ the above conditions are also sufficient.

Let us remark that if both types of Firm 1 were playing mixed strategies $\Gamma$ that is $\Gamma$ randomizing over locations $x_{1}^{* \prime}, x_{1}^{* \prime \prime}, \ldots$, the profits of each type would have to be the same at each one of these locations:

$$
\hat{\Pi}_{1}\left(x_{1}^{* \prime}, \pi, \theta\right)=\hat{\Pi}_{1}\left(x_{1}^{* \prime \prime}, \pi, \theta\right)=\ldots, \theta \in \Theta,
$$

given that $x_{1}^{* \prime}, x_{1}^{* \prime \prime}, \ldots$ are all within the interval $A$ and that $\pi$ satisfies the condition laid down in the last paragraph for each one of these locations. Let us also remark that since $\hat{\Pi}_{1}\left(x_{1}, \pi, \theta\right)$ is concave in $x_{1} \Gamma$ then equation (D3) can be verified for a type $\theta$ by at most two different locations. But if two locations verify (D3) for some type $\theta \Gamma$ then the same locations do not verify (D3) for the other type $\theta^{\prime} \neq \theta$. It is an immediate consequence from Proposition 2. Substitute in (23) $x_{1}^{* \prime}$ for $\dot{x}_{1} \Gamma \pi$ for $\dot{\mu} \Gamma x_{1}^{* \prime}$ for $x_{1}^{\prime} \Gamma x_{1}^{\prime \prime}$ and $\pi$ for $\mu^{\prime}$ and $\mu^{\prime \prime}$. Then it follows from (26) and (27) that if $x_{1}^{* \prime \prime} \neq x_{1}^{* \prime}$ and $\left\{x_{1}^{* \prime \prime}, x_{1}^{* \prime}\right\} \subseteq A \Gamma$ then $\mu\left(x_{1}^{* \prime \prime}, x_{1}^{* \prime}, \pi, c\right) \neq \mu\left(x_{1}^{* \prime \prime}, x_{1}^{* \prime}, \pi, c-\alpha\right) \Gamma$ so that $x_{1}^{* \prime \prime}$ cannot be another equilibrium location. Hence $\Gamma$ pooling equilibria must be pure strategy equilibria.

Let us now show how the D1 refinement may be used in order to eliminate either all the pooling equilibria or all the pooling equilibria but one depending on the values of $\pi$ and $\alpha$. From Cho and Sobel's results we know that in the present model there is for each situation $(\pi, \alpha)$ a unique PBE robust 
to D1. Hence $\Gamma$ since we have already identified for each $\alpha \in\left(0, \hat{\alpha}_{c}\right]$ and each $\pi \in(0,1)$ a separating equilibrium robust to D1 $\mathrm{C}$ no pooling equilibrium survives $\mathrm{D} 1$ in this region. So let us concentrate on the region where $\alpha>\hat{\alpha}_{c}$. In this region $\Gamma$ we have $x_{\max }\left(x_{1}(0, c), 0, c\right)=x_{\max }\left(x_{1}(c-\alpha), 0, c-\alpha\right)=1 / 2$. Let us first show that if a location $\dot{x}_{1}^{*}<1 / 2$ is an equilibrium location $\Gamma$ implying that the prior $\dot{\pi}$ satisfy $\dot{\pi} \geq \max \left\{\mu\left(\dot{x}_{1}^{*}, x_{1}(0, \theta), 0, \theta\right), \theta \in \Theta\right\} \Gamma$ this equilibrium does not survive D1. Consider any alternative location $x_{1}^{\prime} \in\left(\dot{x}_{1}^{*}, x_{\max }\left(\dot{x}_{1}^{*}, \dot{\pi}, c\right)\right)$. From Proposition 2 we know that for such a deviation we have $\mu\left(x_{1}^{\prime}, \dot{x}_{1}^{*}, \dot{\pi}, c-\alpha\right)<\mu\left(x_{1}^{\prime}, \dot{x}_{1}^{*}, \dot{\pi}, c\right)$. Hence observing $x_{1}^{\prime} \Gamma$ Firm 2 should conclude $\Gamma$ according to D $1 \Gamma$ that it is facing the low cost type of Firm 1 . Then from $x_{1}^{\prime}<x_{\max }\left(\dot{x}_{1}^{*}, \dot{\pi}, c\right) \leq x_{\max }\left(\dot{x}_{1}^{*}, \dot{\pi}, c-\alpha\right) \Gamma$ we have $\hat{\Pi}_{1}\left(x_{1}^{\prime}, 1, c-\alpha\right)>\hat{\Pi}_{1}\left(\dot{x}_{1}^{*}, \dot{\pi}, c-\alpha\right)$ sso that Firm 1 of the low cost type will deviate. Last $\Gamma$ let us show that any equilibrium $x_{1}^{*}=1 / 2 \Gamma$ with priors $\pi \geq \max \left\{\mu\left(1 / 2, x_{1}(0, \theta), 0, \theta\right) \theta \in \Theta\right\} \Gamma$ is robust to $\mathrm{D} 1$. For any deviation $x_{1}^{\prime} \in\left[0, x_{\min }\left(x_{1}(0, c), 0, c\right)\right]$ Twe have $\hat{\Pi}_{1}\left(x_{1}^{\prime}, \mu, \theta\right)<\hat{\Pi}_{1}(1 / 2, \pi, \theta), \theta \in \Theta, \mu \in[0,1]$ Thence no type will deviate. For deviation $\left.x_{1}^{\prime} \in\left(x_{\min }(0, c), 0, c\right), 1 / 2\right) \Gamma$ we get from Proposition $2: \mu\left(x_{1}^{\prime}, 1 / 2, \pi, c\right)<\mu\left(x_{1}^{\prime}, 1 / 2, \pi, c-\alpha\right)$. Therefore according to D1 FFirm 2 should conclude that it is facing the high cost type of Firm 1 . Then $\hat{\Pi}_{1}\left(x_{1}^{\prime}, 0, \theta\right) \leq \hat{\Pi}_{1}\left(x_{1}(0, c), 0, \theta\right) \leq \hat{\Pi}_{1}(1 / 2, \pi, \theta) \Gamma \theta \in \Theta \Gamma$ so that no type will deviate.

\section{APPENDIX E: PROOF OF PROPOSITION 5}

In a semi-separating equilibrium $\Gamma$ the supports of the strategies chosen by the two types intersect and for one type at least The support is larger than the intersection.

(E1) Characterization of the set of semi-separating equilibria.

As for separating and pooling equilibria Tany location $x_{1}$ played at equilibrium by the type $\theta, \theta \in \Theta \Gamma$ must be such that $\hat{\Pi}_{1}\left(x_{1}, \mu^{*}\left(c-\alpha \mid x_{1}\right), \theta\right)$ is at least equal to $\hat{\Pi}_{1}\left(x_{1}(0, \theta), 0, \theta\right)$. Hence $\Gamma$ any one of these locations must be within $\bar{A}_{\theta}$ the closure of $A_{\theta}$ defined in (B.7):

$$
\bar{A}_{\theta}=\left[x_{\min }\left(x_{1}(0, \theta), 0, \theta\right), x_{\max }\left(x_{1}(0, \theta), 0, \theta\right)\right] .
$$

However $\Gamma$ if $x_{1}^{*}$ is a location chosen by both types $\Gamma$ then $x_{1}^{*}$ must be within $A_{\theta}$ since the Bayesian posterior belief $\mu^{*}\left(c-\alpha \mid x_{1}^{*}\right)$ is strictly less than 1 :

$$
\mu^{*}\left(c-\alpha \mid x_{1}^{*}\right)=\frac{\pi \nu_{1}\left(x_{1}^{*} \mid c-\alpha\right)}{\pi \nu_{1}\left(x_{1}^{*} \mid c-\alpha\right)+(1-\pi) \nu_{1}\left(x_{1}^{*} \mid c\right)}<1
$$

Suppose now that there exist two locations $x_{1}^{* \prime}$ and $x_{1}^{* \prime \prime}$ both played by the two types of Firm 1 at equilibrium. For these two locations we must have:

$$
\hat{\Pi}_{1}\left(x_{1}^{* \prime}, \mu^{*}\left(c-\alpha \mid x_{1}^{* \prime}\right), \theta\right)=\hat{\Pi}_{1}\left(x_{1}^{* \prime \prime}, \mu^{*}\left(c-\alpha \mid x_{1}^{* \prime \prime}\right), \theta\right), \quad \theta \in \Theta .
$$


But from Proposition 1 it is clearly impossible that this equality be verified for both types. Hence $\Gamma$ in any semi-separating equilibria one and only one location is played by both types.

(E1a) Let us first examine the case where the high cost type is randomizing and the low cost type plays in pure strategy the location $x_{1}^{*}$ also played by the other type.

Consider first the high cost type. Since for any location $x_{1 c}^{*}$ specific to this type at equilibrium the high cost Firm 1 is perfectly identified $\Gamma$ only one such location exists $\Gamma$ the location maximizing the high cost type profit when rightly identified:

$$
x_{1 c}^{*}=x_{1}(0, c) .
$$

The Bayesian posterior belief of Firm 2 observing $x_{1}^{*}$ is equal to:

$$
\mu^{*}\left(c-\alpha \mid x_{1}^{*}\right)=\frac{\pi}{\pi+(1-\pi) \nu_{1}\left(x_{1}^{*} \mid c\right)}<1
$$

Whatever $\mu^{*}\left(c-\alpha \mid x_{1}^{*}\right)<1 \Gamma$ there exists a probability $\nu_{1}\left(x_{1}^{*} \mid c\right) \in(0,1)$ satisfying (E.5) if $\pi<$ $\left.\mu^{*}(c-\alpha) \mid x_{1}^{*}\right)$. The high cost type must be indifferent between $x_{1}^{*}$ and $x_{1 c}^{*}$ :

$$
\hat{\Pi}_{1}\left(x_{1}(0, c), 0, c\right)=\hat{\Pi}_{1}\left(x_{1}^{*}, \mu^{*}\left(c-\alpha \mid x_{1}^{*}\right), c\right)
$$

hence:

$$
x_{1}^{*} \in \bar{A}_{c} \text { and } \mu^{*}\left(c-\alpha \mid x_{1}^{*}\right)=\mu\left(x_{1}^{*}, x_{1}(0, c), 0, c\right) .
$$

Last $\Gamma$ in order that the high cost type does not deviate to any off-the-equilibrium location $x_{1} \notin$ $\left\{x_{1 c}^{*}, x_{1}^{*}\right\}$ Tthe posteriors must verify:

$$
\mu^{*}\left(c-\alpha \mid x_{1}\right) \leq \mu\left(x_{1}, x_{1}(0, c), 0, c\right) .
$$

Consider now the low cost type. In order that it does not deviate from $x_{1}^{*}$ to $x_{1}(0, c)$ Twe must have:

$$
\mu^{*}\left(c-\alpha \mid x_{1}^{*}\right) \geq \mu\left(x_{1}^{*}, x_{1}(0, c-\alpha), 0, c-\alpha\right) \text { and } x_{1}^{*} \in \bar{A}_{c-\alpha} .
$$

Any deviation to an off-the-equilibrium location $x_{1} \neq x_{1}^{*}$ is prevented by posteriors satisfying:

$$
\mu^{*}\left(c-\alpha \mid x_{1}\right) \leq \mu\left(x_{1}, x_{1}^{*}, \mu^{*}\left(c-\alpha \mid x_{1}^{*}\right), c-\alpha\right)
$$

From Proposition 2 and the definition of $\hat{x}_{1}$ as the location $x_{1}$ satisfying $\mu\left(x_{1}, x,(0, c), 0, c\right)=$ $\mu\left(x_{1}, x_{1}(0, c-\alpha), 0, c-\alpha\right)$ Tthe two conditions (E.7) and (E.9) can be met iff $x_{1}^{*} \in\left[\hat{x}_{1}, x_{\max }\left(x_{1}(0, c), 0, c\right)\right)$. In this case all other conditions are satisfied $\Gamma$ provided that $\pi<\mu^{*}\left(c-\alpha \mid x_{1}^{*}\right)=\mu\left(x_{1}^{*}, x_{1}(0, c), 0, c\right)$. 
(E1b) Let us examine now the equilibria where the low cost type randomizes and the high cost type plays a pure strategy $x_{1}^{*}$.

Consider first the low cost type. The common location $x_{1}^{*}$ and all its specific locations $x_{1 c-\alpha}^{* \prime}, x_{1 c-\alpha}^{* \prime \prime}, \ldots$ must be within $\bar{A}_{c-\alpha}$ :

$$
x_{1}^{*}, x_{1 c-\alpha}^{* 1}, x_{1 c-\alpha}^{* \prime \prime}, \ldots, \in \bar{A}_{c-\alpha} .
$$

The posterior of Firm 2 observing $x_{1}^{*}$ now takes the following form:

$$
\mu^{*}\left(c-\alpha \mid x_{1}^{*}\right)=\frac{\pi \nu_{1}\left(x_{1}^{*} \mid c-\alpha\right)}{\pi \nu_{1}\left(x_{1}^{*} \mid c-\alpha\right)+(1-\pi)}<1 .
$$

For any given $\mu^{*}\left(c-\alpha \mid x_{1}^{*}\right)<1 \Gamma$ there exists a randomization $\nu_{1}\left(x_{1}^{*} \mid c-\alpha\right)$ satisfying (E.12) iff $\pi>\mu^{*}\left(c-\alpha \mid x_{1}^{*}\right)$. Since all the locations give to the type $c-\alpha$ the same profit $\Gamma$ then:

$$
\hat{\Pi}_{1}\left(x_{1}^{*}, \mu^{*}\left(c-\alpha \mid x_{1}^{*}\right), c-\alpha\right)=\hat{\Pi}_{1}\left(x_{1 c-\alpha}^{* \prime}, 1, c-\alpha\right)=\hat{\Pi}_{1}\left(x_{1 c-\alpha}^{* \prime \prime}, 1, c-\alpha\right)=\ldots
$$

Hence $\Gamma$ there may be at most two specific locations $\Gamma$ the first one being $x_{1 c-\alpha}^{* \prime}<\min \left\{x_{1}^{*}, x_{1}(1, c-\alpha)\right\}$. Note that if $\alpha \geq 1 / 8 \Gamma x_{\max }\left(x_{1}(0, c-\alpha), 0, c-\alpha\right)=x_{1}(1, c-\alpha) \Gamma$ so that for those values of $\alpha$ there exists only one specific location $x_{1 c-\alpha}^{*}<\min \left\{x_{1}^{*}, x_{1}(1, c-\alpha)\right\}$. (E.11) and (E.13) imply that:

$$
\begin{gathered}
\mu^{*}\left(c-\alpha \mid x_{1}^{*}\right)=\mu\left(x_{1}^{*}, x_{1 c-\alpha}^{* \prime}, 1, c-\alpha\right)=\mu\left(x_{1}^{*}, x_{1 c-\alpha}^{* \prime \prime}, 1, c-\alpha\right) \\
x_{1}^{*} \in\left[x_{\min }\left(x_{1 c-\alpha}^{*}, 1, c-\alpha\right), x_{\max }\left(x_{1 c-\alpha}^{*}, 1, c-\alpha\right)\right]
\end{gathered}
$$

where $x_{1 c-\alpha}^{*}$ is either the unique specific location or any one of them if two such locations exist. Last $\Gamma$ for any off-the-equilibrium location $x_{1}$ Tthe following condition must hold:

$$
\mu^{*}\left(c-\alpha \mid x_{1}\right) \leq \mu\left(x_{1}, x_{1}^{*}, \mu^{*}\left(c-\alpha \mid x_{1}^{*}\right), c-\alpha\right)
$$

For the high cost typeTwe must first have:

$$
x_{1}^{*} \in \bar{A}_{c} \text { and } \mu^{*}\left(c-\alpha \mid x_{1}^{*}\right) \geq \mu\left(x_{1}^{*}, x,(0, c), 0, c\right) .
$$

If not $\Gamma$ the high cost type would deviate from $x_{1}^{*}$ to $x_{1}(0, c)$. For any off-the-equilibrium $x_{1}$ the posteriors must be such that:

$$
\mu^{*}\left(c-\alpha \mid x_{1}\right) \leq \mu\left(x_{1}, x_{1}^{*}, \mu^{*}\left(c-\alpha \mid x_{1}^{*}\right), c-\alpha\right) .
$$

Finally Tadditional conditions must be satisfied in order that the high cost type does not mimic the low cost type:

$$
\hat{\Pi}_{1}\left(x_{1 c-\alpha}^{* \prime}, 1, c\right) \leq \hat{\Pi}_{1}\left(x_{1}^{*}, \mu^{*}\left(c-\alpha \mid x_{1}^{*}\right), c\right)
$$




$$
\hat{\Pi}_{1}\left(x_{1 c-\alpha}^{* \prime \prime}, 1, c\right) \leq \hat{\Pi}_{1}\left(x_{1}^{*}, \mu^{*}\left(c-\alpha \mid x_{1}^{*}\right), c\right) .
$$

Therefore The specific location $x_{1 c-\alpha}^{* \prime}<x_{1}(1, c-\alpha)$ must satisfy:

$$
x_{1 c-\alpha}^{* \prime}<x_{\min }\left(x_{1}^{*}, \mu^{*}\left(c-\alpha \mid x_{1}^{*}\right), c\right)
$$

implying that:

$\mu\left(x_{\min }\left(x_{1}^{*}, \mu^{*}\left(c-\alpha \mid x_{1}^{*}\right), c\right), x_{1}^{*}, \mu^{*}\left(c-\alpha \mid x_{1}^{*}\right), c-\alpha\right)<\mu\left(x_{\min }\left(x_{1}^{*}, \mu^{*}\left(c-\alpha \mid x_{1}^{*}\right), c\right), x_{1}^{*}, \mu^{*}\left(c-\alpha \mid x_{1}^{*}\right), c\right)=1$.

But from Proposition 2 (with $\dot{x}_{1}=x_{1}^{\prime}=x_{1}^{\prime \prime}=x^{*}$ Tand $\dot{\mu}=\mu^{\prime}=\mu^{\prime \prime}=\mu^{*}\left(c-\alpha \mid x_{1}^{*}\right)<1$ in (23) and $x_{1}=x_{1-c}^{* \prime}$ in (26)) the inequality (E.20) would have to be in the opposite sense. Hence $\mathrm{C}$ there may exist only one specific location which we will denote by $x_{1 c-\alpha}^{*}$ and such that $x_{1 c-\alpha}^{*}>x_{1}(1, c-\alpha) \Gamma$ implying that $\alpha$ must be less than $1 / 8$. Now let $x_{1 c-\alpha}^{*} \in\left(x_{1}(1, c-\alpha), x_{\max }\left(x_{1}(0, c), 0, c\right)\right]$. Then from Proposition 2 (with $\dot{\mu}=1=\mu^{*}\left(c-\alpha \mid x_{1 c-\alpha}^{*}\right.$ ) and $\dot{x}_{1}=x_{1 c-\alpha}^{*}$ in (25) and $x_{1}=x_{1}^{*}$ in (26)) Гwe get for any $x_{1}^{*} \in\left(x_{\min }\left(x_{1 c-\alpha}^{*}, 1, c\right), x_{1 c-\alpha}^{*}\right)$ :

$$
\mu\left(x_{1}^{*}, x_{1 c-\alpha}^{*}, 1, c\right)<\mu\left(x_{1}^{*}, x_{1 c-\alpha}^{*}, 1, c-\alpha\right) .
$$

Hence $\Gamma$ if $\nu_{1}\left(x_{1}^{*} \mid c-\alpha\right)$ and $\pi$ are such that:

$$
\mu^{*}\left(c-\alpha \mid x_{1}^{*}\right)=\mu\left(x_{1}^{*}, x_{1 c-\alpha}^{*}, 1, c-\alpha\right)
$$

then:

- the high cost type does not mimic the low cost type (by (E.23)) Гand $\Gamma$

- since $x_{1 c-\alpha}^{*}<x_{\max }\left(x_{1}(0, c), 0, c\right) \Gamma$ then $\mu\left(x_{1 c-\alpha}^{*}, x_{1}(0, c), 0, c\right)<\mu\left(x_{1 c-\alpha}^{*}, x_{1 c-\alpha}^{*}, 1, c\right)=1 \Gamma$ implying that $\mu\left(x_{1}^{*}, x_{1}(0, c), 0, c\right)<\mu\left(x_{1}^{*}, x_{1 c-\alpha}^{*}, 1, c\right)<\mu\left(x_{1}^{*}, x_{1 c-\alpha}^{*}, 1, c-\alpha\right)=\mu^{*}\left(c-\alpha \mid x_{1}^{*}\right) \Gamma$ so that (E.17) is also satisfied.

Last $\Gamma$ suppose that $\alpha \in\left(0, \hat{\alpha}_{c}\right] \Gamma$ so that $x_{\max }\left(x_{1}(0, c), 0, c\right) \leq x_{\max }\left(x_{1}(0, c-\alpha), 0, c-\alpha\right)$ (with the strict inequality if $\left.x<\alpha_{c}\right)$ and consider a location $x_{1 c-\alpha}^{*} \in\left(x_{\max }\left(x_{1}(0, c), 0, c\right), x_{\max }\left(x_{1}(0, c-\alpha), 0, c-\right.\right.$ $\alpha)$. In (23) $\Gamma$ let $\dot{x}_{1}=\bar{x}_{1}\left(x_{1 c-\alpha}^{*}\right), x_{1}^{\prime}=x_{1}(0, c), x_{1}^{\prime \prime}=x_{1}(0, c-\alpha)$ and $\dot{\mu}=\mu\left(x_{1}^{*}, x_{1}(0, c), 0, c\right)=$ $\mu\left(x_{1}^{*}, x_{1 c-\alpha}^{*}, 1, c-\alpha\right), \mu^{\prime}=0, \mu^{\prime \prime}=0$. From Proposition $2 \Gamma$ we get for any $x_{1}^{*}$ satisfying (E.11) and (E.18):

$\mu\left(x_{1}^{*}, \bar{x}_{1}\left(x_{1 c-\alpha}^{*}\right), \dot{\mu}, c\right) \leq(>) \mu\left(x_{1}^{*}, \bar{x}_{1}\left(x_{1 c-\alpha}^{*}\right), \dot{\mu}, c-\alpha\right)=\mu\left(x_{1}^{*}, x_{1 c-\alpha}^{*}, 1, c-\alpha\right)$ iff $x_{1}^{*} \leq(>) \bar{x}_{1}\left(x_{1 c-\alpha}^{*}\right)$. 
Hence $\Gamma$ if $x_{1}^{*} \leq \bar{x}_{1}\left(x_{1 c-\alpha}^{*}\right)$ and $\pi>\mu\left(x_{1}^{*}, \bar{x}_{1}\left(x_{1 c-\alpha}^{*}\right), \dot{\mu}, c-\alpha\right)$ Tthen $\nu_{1}\left(x_{1}^{*} \mid c-\alpha\right)$ may be chosen so that:

$$
\mu^{*}\left(x_{1}^{*} \mid c-\alpha\right)=\mu\left(x_{1}^{*}, x_{1 c-\alpha}^{*}, 1, c-\alpha\right)
$$

ThusTrecapitulating:

$$
\mu^{*}\left(c-\alpha \mid x_{1}^{*}\right)=\mu\left(x_{1}^{*}, \bar{x}_{1}\left(x_{c-\alpha}^{*}\right), 1, c-\alpha=\mu\left(x_{1}^{*}, x_{1 c-\alpha}^{*}, 1, c \alpha\right)>\mu\left(x_{1}^{*}, x,(0, c), 0, c\right) .\right.
$$

Hence (E.17) is satisfied. Since $x_{1 c-\alpha}^{*} \geq x_{\max }\left(x_{1}(0, c), 0, c\right) \Gamma$ the high cost type does not mimic the

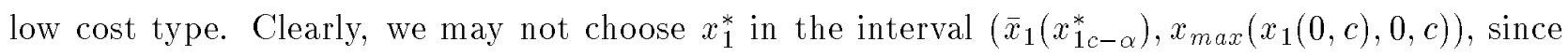
by (E.25) we would have $\mu\left(x_{1}^{*}, x_{1}(0, c), 0, c\right)>\mu\left(x_{1}^{*}, x_{1 c-\alpha}^{*} 1, c-\alpha\right) \Gamma$ so that both (E.14) and (E.17) would not be satisfied.

(E1c) Last Tconsider the equilibria when both types randomize.

For the high cost type there may only be one specific location:

$$
x_{1 c}^{*}=x_{1}(0, c)
$$

and the common location must satisfy:

$$
x_{1}^{*} \in \bar{A}_{c} \text { and } \mu^{*}\left(c-\alpha \mid x_{1}^{*}\right)=\mu\left(x_{1}^{*}, x_{1}(0, c), 0, c\right) .
$$

For the low cost type there may be at most two specific locations the common one the profit must be the same:

$$
x_{1 c-\alpha}^{* \prime}=x_{\min }\left(x_{1}^{*}, \mu^{*}\left(c-\alpha \mid x_{1}^{*}\right), c-\alpha\right) \text { and } x_{1 c-\alpha}^{* \prime \prime}=x_{\max }\left(x_{1}^{*}, \mu^{*}\left(c-\alpha \mid x_{1}^{*}\right), c-\alpha\right)
$$

with:

$$
x_{1}^{*}, x_{1 c-\alpha}^{* \prime}, x_{1 c-\alpha}^{* \prime \prime} \in \bar{A}_{c-\alpha} .
$$

Note that in the present case $\mu^{*}\left(c-\alpha \mid x_{1}^{*}\right)$ is given by:

$$
\mu^{*}\left(c-\alpha \mid x_{1}^{*}\right)=\frac{\pi \nu_{1}\left(x_{1}^{*} \mid c-\alpha\right)}{\pi \nu_{1}\left(x_{1}^{*} \mid c-\alpha\right)+(1-\pi) \nu_{1}\left(x_{1}^{*} \mid c\right)}
$$

so that whatever $\pi$ There exists a whole range of randomizations $\left\{\nu_{1}\left(x_{1}^{*} \mid c-\alpha\right), \nu_{1}\left(x_{1}^{*} \mid c\right)\right\}$ satisfying (E.32).

Let us now examine the self-selection constraint for the high cost type. In order that the high cost type does not deviate from either $x_{1}(0, c)$ or $x_{1}^{*}$ to either $x_{1 c-\alpha}^{* \prime}$ or $x_{1 c-\alpha}^{* \prime \prime}$ T these last two locations must be: - outside $\left(x_{\min }\left(x_{1}(0, c), 0, c\right), x_{\max }\left(x_{1}(0, c), 0, c\right)\right)$ if $\alpha<\hat{\alpha}_{c} \Gamma$ since for such values of $\alpha \Gamma$ 
$x_{\max }\left(x_{1}(0, c), 0, c\right) \leq 1 / 2 ;-$ outside $\left(x_{\min }\left(x_{1}(0, c), 0, c\right), 1 / 2\right]$ if $\alpha \geq \hat{\alpha}_{c} \Gamma$ since for such values of $\alpha \Gamma$ $x_{\max }\left(x_{1}(0, c), 0, c\right)=1 / 2$

and $\mu\left(1 / 2, x_{1}(0, c), 0, c\right) \leq 1$ (the strict inequality if $\left.\alpha>\hat{\alpha}_{c}\right)$. Hence $\mathrm{from} x_{1 c-\alpha}^{* \prime} \geq x_{\min }\left(x_{1}(0, c-\right.$ $\alpha), 0, c-\alpha)>x_{\min }\left(x_{1}(0, c), 0, c\right)$ (the first inequality form $\left.(\mathrm{E} .31)\right) \Gamma$ we conclude that the candidate specific location $x_{1 c-\alpha}^{* \prime}$ cannot meet the constraints $\Gamma$ whatever $\alpha$. We are thus left with only one candidate specific location $x_{1 c-\alpha}^{*}=x_{\max }\left(x_{1}^{*}, \mu^{*}\left(c-\alpha \mid x_{1}^{*}\right), c-\alpha\right)$. However $\Gamma$ if $\alpha>\hat{\alpha}_{c-\alpha}$ then $x_{\max }\left(x_{1}(0, c-\alpha), 0, c-\alpha\right)=1 / 2=x_{\max }\left(x_{1}(0, c), 0, c\right) \Gamma$ so that the only remaining candidate is also eliminated. Hence: - if $\alpha>\hat{\alpha}_{c}$ T there exists no semi-separating equilibrium where both types randomize; - if $\alpha \leq \hat{\alpha}_{c}$ Tthe low cost type randomizes over two and only two locations:

$$
x_{1}^{*} \text { and } x_{1 c-\alpha}^{*}=x_{\max }\left(x_{1}^{*}, \mu^{*}\left(c-\alpha \mid x_{1}^{*}\right), c-\alpha\right) .
$$

Now let us suppose that $\alpha<\hat{\alpha}_{c-\alpha}$ implying that $x_{\max }\left(x_{1}(0, c), 0, c\right)<x_{\max }\left(x_{1}(0, c-\alpha), 0, c-\alpha\right) \leq 1 / 2$ (the inequality strict if $\alpha<\hat{\alpha}_{c-\alpha}$ ) (see Figure 3 ). From Proposition $2 \Gamma$ we deduce that in order that (E.7) and (E.33) be satisfied $\Gamma$ it is necessary that:

$$
x_{1}^{*} \in\left[\hat{x}_{1}, x_{\max }\left(x_{1}(0, c), 0, c\right)\right) .
$$

Figure 3 about here

Again $\Gamma$ applying Proposition 2 (with $\dot{x}_{1}=x_{1}^{*}=x_{1}^{\prime}=x_{1}^{\prime \prime}$ and $\dot{\mu}=\mu\left(x_{1}^{*}, x_{1}(0, c), 0, c\right)=\mu^{\prime}=\mu^{\prime \prime}$ in (23)) $\Gamma$ we know that for $x_{1} \in\left(x_{1}^{*}, x_{\max }\left(x_{1}^{*}, \mu^{*}\left(c-\alpha \mid x_{1}^{*}\right), c-\alpha\right)\right)$ then $\mu\left(x_{1}, x_{1}^{*}, \mu^{*}\left(c-\alpha \mid x_{1}^{*}\right), c\right)>$ $\mu\left(x_{1}, x_{1}^{*}, \mu^{*}\left(c-\alpha \mid x_{1}^{*}\right), c-\alpha\right)$. Hence $\operatorname{cby}$ continuity $\mu\left(x_{\max }\left(x_{1}^{*}, \mu^{*}\left(c-\alpha \mid x_{1}^{*}\right), c-\alpha\right), x_{1}^{*}, \mu^{*}\left(c-\alpha \mid x_{1}^{*}\right), c\right) \geq$ $\mu\left(x_{\max }\left(x_{1}^{*}, \mu^{*}\left(c-\alpha \mid x_{1}^{*}\right), c-\alpha\right), x_{1}^{*}, \mu^{*}\left(c-\alpha \mid x_{1}^{*}\right), c-\alpha\right) \Gamma$ so that the high cost type is not incited to switch to the low cost specific location $x_{1 c-\alpha}^{*}=x_{\max }\left(x_{1}^{*}, \mu^{*}\left(c-\alpha \mid x_{1}^{*}\right), c-\alpha\right)$. Also $\Gamma$ since $x_{1}^{*} \in$ $\left[\hat{x}_{1}, x_{\max }\left(x_{1}(0, c), 0, c\right)\right)$ then $x_{1 c-\alpha}^{*}=x_{\max }\left(x_{1}^{*}, \mu^{*}\left(c-\alpha \mid x_{1}^{*}\right), c-\alpha\right) \in\left[x_{\max }\left(x_{1}(0, c), 0, c\right), x_{\max }\left(x_{1}(0, c-\right.\right.$ $\alpha), 0, c-\alpha)$ ]. Last $\Gamma$ suppose that $\alpha \in\left(\hat{\alpha}_{c-\alpha}, \hat{\alpha}_{c}\right]$ so that $x_{\max }\left(x_{1}(0, c), 0, c\right) \leq x_{\max }\left(x_{1}(0, c-\alpha), 0, c-\right.$ $\alpha)=1 / 2\left(\right.$ the first inequality strict if $\left.\alpha<\hat{\alpha}_{c}\right)$. See Figure 4.

Figure 4 about here

For any $x_{1}^{*} \in\left[\ddot{x}_{1}, x_{\max }\left(x_{1}(0, c), 0, c\right)\right)$ we have:

$$
x_{\max }\left(x_{1}^{*}, \mu\left(x_{1}^{*}, x_{1}(0, c), 0, c\right), c-\alpha\right) \in\left[x_{\max }\left(x_{1}(0, c), 0, c\right), 1 / 2\right]
$$

and

$$
\mu\left(x_{\max }\left(x_{1}^{*}, \mu\left(x_{1}^{*}, x_{1}(0, c), 0, c\right), x_{1}^{*}, \mu\left(x_{1}^{*}, x_{1}(0, c), 0, c\right), c-\alpha\right)=1\right.
$$

and for any $x_{1}^{*} \in\left[\hat{x}_{1}, \ddot{x}_{1}\right)$ :

$$
x_{\max }\left(x_{1}^{*}, \mu\left(x_{1}^{*}, x_{1}(0, c), 0, c\right), c-\alpha\right)<1 / 2
$$


and

$$
\mu\left(1 / 2, x_{1}^{*}, \mu\left(x_{1}^{*}, x_{1}(0, c), 0, c\right), c-\alpha\right)<1 .
$$

Hence in this last case the high cost type would mimic the low cost type. We conclude that the common location $x_{1}^{*}$ must be within $\left[\ddot{x}_{1}, x_{\max }\left(x_{1}(0, c), 0, c\right)\right)$.

(E2) The working of the D1 criterion.

We know from Cho and Sobel's results that no semi-separating equilibrium survives D1 in regions where we have already shown that either a separating equilibrium or a pooling equilibrium survives D1. Therefore $\Gamma$ we may restrict the analysis to the region defined by $\alpha>\hat{\alpha}_{c}$ and $\pi<$ $\mu\left(1 / 2, x_{1}(0, c), 0, c\right)$. Consider in this region the equilibria in which only the high cost Firm 1 randomizes over the two locations $x_{1 c}^{*}=x_{1}(0, c)$ and $x_{1}^{*}=1 / 2$. For any $x_{1 c}^{\prime} \in\left(x_{\min }\left(x_{1}(0, c), 0, c\right), 1 / 2\right) \Gamma$ we get from Proposition 2:

$$
\mu\left(x_{1}^{\prime}, 1 / 2, \mu^{*}(c-\alpha \mid 1 / 2), c\right)<\mu\left(x_{1}^{\prime}, 1 / 2, \mu^{*}(c-\alpha \mid 1 / 2), c-\alpha\right) .
$$

Hence observing $x_{1}^{\prime}$ TFirm 2 should conclude $\Gamma$ according to D1T that it is facing the high cost type of Firm 1. Therefore neither the high cost type nor the low cost type would gain more than its equilibrium profit. For deviations $x_{1}^{\prime} \in\left[0, x_{\min }\left(x_{1}(0, c), 0, c\right)\right] \Gamma$ no type would benefit from the deviation $\Gamma$ whatever the posteriors of Firm 2 observing the deviation. We conclude that for the region under consideration $\Gamma$ we have identified the unique D1 equilibrium. 


\section{References}

[1] BoyerГМ.ГLaffontГJ.J. MahencГР.ГМоreauxГМ.: Location distortions under incomplete information. Reg. Sci. Urban Econ.Г24Г409-440 (1994)

[2] Carlton TD.W.: A reexamination of delivered pricing systems. J. Law Econ. 26 Г51-70 (1983)

[3] ChoГІ.К.ГSobelГJ.: Strategic stability and uniqueness in signaling games. J. Econ. Theory 50Г381$413(1990)$

[4] D’AspremontГС.ГJaskold-GabszewiczГЈ.ГТhisseГJ.F.: On Hotelling stability in competition. Econometrica $47 \Gamma 1145-1150(1979)$

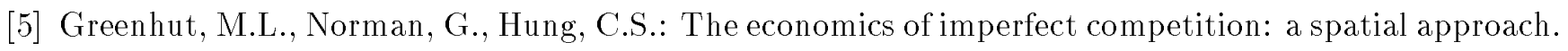
Cambridge: Cambridge University Press 1987

[6] Hobbs[ B.F.: Mill pricing versus spatial price discrimination under Bertrand and Cournot spatial competition. J. Ind. Econ. 35 173-191 (1986)

[7] HooverГE.M. Jr.: Spatial price discrimination. Rev. Econ. Studies 4Г182-191 (1937)

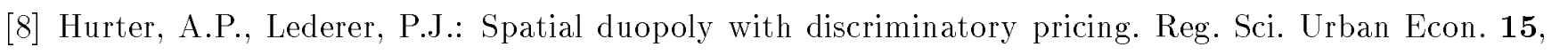
$541-553(1985)$

[9] Jaskold-GabszewiczГ J.ГThisseГ J.F.: Spatial competition and the location of firms. in LesourneГ J.Г SonnenscheinГH. (eds.): Fundamentals of pure and applied economics. London: Harwood 1986

[10] Jaskold-GabszewiczГJ.ГThisseГJ.F.: Location. in AumannГR.J.ГHartГSГ (eds): Handbook of game theory with economic applications. Amsterdam: North-Holland 1992

[11] LedererГР.J.ГHurterГА.P.: Competition of firms: discriminatory pricing and location. Econometrica 54Г623-640 (1986)

[12] MilgromГ Р.Г RobertsГ J.: Limit pricing and entry under incomplete information: an equilibrium analysis. Econometrica 50Г443-459 (1982)

[13] ThisseГJ.F.ГVivesГХ.: On the strategic choice of spatial price policy. Amer. Econ. Rev. 78Г122-137 (1988) 